\title{
Sox2 haploinsufficiency primes regeneration and Wnt responsiveness in the mouse cochlea
}

\author{
Patrick J. Atkinson, ${ }^{1}$ Yaodong Dong, ${ }^{1,2}$ Shuping Gu, ${ }^{1}$ Wenwen Liu, ${ }^{1}$ Elvis Huarcaya Najarro, ${ }^{1}$ Tomokatsu Udagawa, ${ }^{1}$ \\ and Alan G. Cheng ${ }^{1}$
}

'Department of Otolaryngology - Head and Neck Surgery, Stanford University School of Medicine, Stanford, California, USA. Department of Otology, Shengjing Hospital of China Medical University, Shenyang, Liaoning, China.

\begin{abstract}
During development, Sox2 is indispensable for cell division and differentiation, yet its roles in regenerating tissues are less clear. Here, we used combinations of transgenic mouse models to reveal that Sox 2 haploinsufficiency (Sox $2^{\text {haplo }}$ ) increases rather than impairs cochlear regeneration in vivo. Sox $2{ }^{\text {haplo }}$ cochleae had delayed terminal mitosis and ectopic sensory cells, yet normal auditory function. Sox $2^{\text {haplo }}$ amplified and expanded domains of damage-induced Atoh1 ${ }^{+}$transitional cell formation in neonatal cochlea. Wnt activation via $\beta$-catenin stabilization ( $\beta$-catenin ${ }^{\mathrm{COF}}$ ) alone failed to induce proliferation or transitional cell formation. By contrast, $\beta$-catenin ${ }^{\mathrm{COF}}$ caused proliferation when either Sox $2^{\text {haplo }}$ or damage was present, and transitional cell formation when both were present in neonatal, but not mature, cochlea. Mechanistically, Sox $2^{\text {haplo }}$ or damaged neonatal cochleae showed lower levels of Sox2 and Hes5, but not of Wnt target genes. Together, our study unveils an interplay between Sox2 and damage in directing tissue regeneration and Wnt responsiveness and thus provides a foundation for potential combinatorial therapies aimed at stimulating mammalian cochlear regeneration to reverse hearing loss in humans.
\end{abstract}

\section{Introduction}

SRY-box 2 (Sox2) is a SoxB1 HMG domain transcription factor critical for establishing and maintaining the pluripotency of embryonic stem cells $(1,2)$ and, in combination with other defined factors, induces pluripotent stem cells $(3,4)$. Sox 2 also directs cellular differentiation in multiple developing organs, including the CNS (5-9), retina (10), cochlea (11), hair follicles (12), lens (13), and the foregut and its derivatives (14-17). Besides designating tissuespecific progenitors in these developing organs, Sox 2 governs cell specification $(10,11,17$, with deficiencies leading to anophthalmia and epilepsy as well as trachea-esophageal and genital anomalies in both mice and humans (10, 17-20).

Sox2-expressing somatic stem cells are widely distributed in adult animals and are essential for tissue homeostasis and repair $(16,21-23)$. For example, Sox 2 loss of function impairs adult neurogenesis and tracheal repair $(16,24-26)$. Moreover, Sox 2 is upregulated during spinal cord regeneration, with damage inducing the proliferation of Sox $2^{+}$cells, and inhibition of Sox 2 limiting their regeneration in a dose-dependent manner (27). Thus, the prevailing notion is that Sox 2 insufficiency impairs both the development and regeneration of tissues.

In the developing mouse cochlea, Sox 2 expression marks the prosensory region, which harbors cells primed to give rise to the mechanosensory hair cells and nonsensory supporting cells of the organ of Corti. Sox 2 is required for hair cell specification, as pro-

Authorship note: PJA and YD contributed equally to this work. Conflict of interest: The authors have declared that no conflict of interest exists. Submitted: September 1, 2017; Accepted: February 1, 2018. Reference information: / Clin Invest. 2018;128(4):1641-1656. https://doi.org/10.1172/JCI97248 sensory cells in Sox2-deficient cochleae fail to acquire a hair cell fate $(11,28)$. A subset of Sox $2^{+}$cells first express the prosensory transcription factor Atoh1 around E13.5 (29). Specified sensory cells expressing Atoh1 then mature by upregulating Pou4f3, a transcription factor linked with inherited progressive hearing loss in humans $(30,31)$, as well as Gfi1 (32) and myosin 7a (33). Conversely, Sox 2 is downregulated in maturing hair cells and becomes undetectable in the early postnatal period, while adjacent supporting cells maintain Sox2 expression (34). By binding to its enhancer region, Sox 2 can directly activate Atoh1 (35-38) and is sufficient for inducing ectopic sensory hair cells in vitro (38) and in vivo (39) in the embryonic cochlea. A subsequent reduction of Sox 2 expression is also necessary for hair cell maturation, as high levels of Sox 2 antagonize Atoh1 and prevent hair cell formation when overexpressed in vitro $(28,35)$, and Sox 2 activates repressors of Atoh1 (40). Because Sox2 is initially required for hair cell specification and subsequently inhibits hair cell differentiation, its relationship with hair cell formation is coined an "incoherent feed-forward loop" (41).

Here, we examined the effects of Sox 2 haploinsufficiency on cell division and hair cell regeneration in response to damage in the postnatal cochlea. We found that Sox2-haploinsufficient mice had extranumerary cochlear hair cells and normal auditory function in adulthood. Although terminal mitosis normally occurs around E14.5 (42), supporting cells in the postnatal Sox2haploinsufficient cochlea still divided. After hair cell ablation in the neonatal cochlea, supporting cells that proliferate and form new hair cells are limited in number and spatially restricted (43). In the damaged Sox2-haploinsufficient cochlea, we found an increase in and expansion of the domain of dividing $\left(\mathrm{EdU}^{+}\right)$and transitional $\left(\right.$ Atoh $^{+}$) supporting cells along the cochlea. Moreover, 
Sox2 haploinsufficiency and damage act as permissive signals to $\beta$-catenin-induced proliferation and formation of transitional cells (cells undergoing a direct phenotypic conversion from supporting cells to hair cell-like cells) in neonatal, but not mature, cochlea. Together, our results show cooperative roles of Sox2 haploinsufficiency and Wnt signaling in regulating the spatiotemporally restricted regenerative responses - proliferation and transitional cell formation - in the postnatal mouse cochlea.

\section{Results}

Sox2 haploinsufficiency leads to ectopic hair cell formation and delayed terminal mitosis. The mammalian cochlea is organized as a checkerboard of hair cells intercalated by Sox $2^{+}$supporting cells (34) (Figure $1 \mathrm{~A})$. In the $\mathrm{P} 5$ cochlea, Sox 2 expression was robust in most supporting cell subtypes (Hensen's cells, Deiters' cells, pillar cells, and inner phalangeal cells) and also in cells residing in the greater epithelial ridge (Figure 1A). This pattern was confirmed by Cre reporter expression in Sox $2^{\text {CreERT2/+ }}$ Rosa26 $R^{\text {TdTomato/+ }}$ cochleae induced on P1 and by GFP expression in Sox $2^{\text {GFP/+ }}$ cochleae (Figure 1, B and C) (21).

To determine whether hair cell formation and proliferation are affected by Sox 2 haploinsufficiency, we first examined cochleae from Sox $2^{\mathrm{CrEERT2/+}}$ mice (Figure 1D). The Sox $2^{\mathrm{CrERRT2/+}}$ mouse was generated as an inserted targeted mutation in the single exon of the Sox2 gene (21), resulting in Sox2 haploinsufficiency (Sox $2^{\text {haplo }}$ ). We performed quantitative PCR (qPCR) on cochleae from P5 Sox $2^{\text {CreERT2/+ }}$ mice and found a reduction of approximately $27.3 \%$ in Sox 2 expression relative to WT cochleae (Figure 1E, $P<0.05$ ). P5 WT cochleae had the normal complement of myosin $7 \mathrm{a}^{+}$hair cells ( 3 rows of outer hair cells and 1 row of inner hair cells) (Figure $1 \mathrm{~F}$ ). In Sox $2^{\text {CreERT2/+ }}$ cochleae, we noted extranumerary myosin $7 \mathrm{a}^{+}$hair cells juxtaposed to inner hair cells (Figure $1 G$ ) along the length of the cochlea. We also observed ectopic hair cells along the cochleae from a second Sox2-knockin mouse line (Sox $2^{\text {GFP/+ }}$ ) (Supplemental Figure 1, B-E; supplemental material available online with this article; https:// doi.org/10.1172/JCI97248DS1) (21). On average, we observed 78.2 \pm 38.1 and $65.5 \pm 27.0$ ectopic hair cells in whole cochleae from So $2^{\text {CreERT2/+ }}$ and Sox $2^{\text {GFP/+ }}$ mice, respectively, compared with $3.3 \pm 1.5$ ectopic hair cells in WT control cochleae (Figure $1 \mathrm{H})$.

The last mitotic event in the developing organ of Corti occurs in the basal turn around E14.5 (42). EdU pulses (P2-P4, Figure 1D) failed to label any hair cells or supporting cells in the WT cochlea, confirming its mitotic quiescence (Figure $1 \mathrm{~F}$ ). With the same EdU regimen (Figure 1D and Supplemental Figure 1A), we observed $10.2 \pm 4.8$ and $22.8 \pm 13.8$ EdU-labeled supporting cells in the apical turn of Sox $2^{\mathrm{CrEERT2} /+}$ and Sox $2^{\mathrm{GFP} /+}$ cochleae, respectively (Figure 1, G and I, Supplemental Figure 1C, and Supplemental Table 1). There were no EdU ${ }^{+}$supporting cells in the middle or basal turns (Supplemental Figure 1, D and E). To determine the timing of terminal mitosis in Sox $2^{\text {CreERT2/+ }}$ mice, we delayed the EdU injection schedule by 1 day (P3-P5) and failed to detect any EdU-labeled supporting cells in the organ of Corti ( $n=3$, data not shown). This indicates that terminal mitosis is delayed until around P2 in the So $x 2^{\text {CreERT2/+ }}$ cochlea. We confirmed this finding by immunostaining for the proliferation marker Ki67 in Sox $2^{\text {CreERT2/+ }}$ and Sox $2^{\text {GFP/+ }}$ cochleae (P4-P5) and found no Ki67 ${ }^{+}$cells in the organ of Corti (Supplemental Figure 1, F-H). We also measured the auditory brainstem response (ABR) and distortion product otoacous- tic emissions (DPOAEs) and found no statistical differences in thresholds between Sox $2^{\text {CreERT2/+ }}$ mice and WT mice at 4 weeks of age (Figure 1J and Supplemental Figure 1I). These results demonstrate that Sox2 haploinsufficiency (Sox $2^{\text {haplo }}$ ) leads to delayed terminal mitosis and ectopic inner hair cell formation without compromising auditory function.

So $2^{\text {haplo }}$ enhances mitotic regeneration and transitional cell formation. Our prior work showed that supporting cells in the apical turn mitotically regenerate hair cells after hair cell ablation in the neonatal cochlea (43). Here, we compared damaged (Pou $\left.4 \mathrm{f}^{\mathrm{DTR} /+}\right)$ (44) cochleae with damaged, Sox $2^{\text {haplo }}$ (Pou $4 f^{\text {DTR/+ }}$ Sox $2^{\text {CreERT2/+ }}$ ) cochleae (Figure 2A and Supplemental Figure 3E). Cochleae from WT animals injected with diphtheria toxin (DT) showed no hair cell loss or EdU-labeled supporting cells (Figure 2, B-D). As expected, DTinduced hair cell loss in Pou $4 \mathrm{f}^{\mathrm{DTR} /+}$ mice resulted in a modest increase in EdU ${ }^{+}$hair cells $\left(0.3 \pm 0.1\right.$ per $160 \mu \mathrm{m}$, myosin $\left.7 \mathrm{a}^{+}\right)$and supporting cells $\left(1.4 \pm 0.5\right.$ per $160 \mu \mathrm{m}$, Sox $2^{+}$myosin $\left.7 \mathrm{a}^{-}\right)$in the apical turn (Figure 2E and Supplemental Table 1). We found no proliferative cells in the middle or basal turns of the damaged cochlea (Figure 2, F and G). In the Pou $43^{\text {DTR/+ }}$ Sox $2^{\text {CreERT2/+ }}$ (damaged, Sox $2^{\text {haplo) }}$ cochlea, we found a significantly larger number of EdU-labeled supporting cells $(10.3 \pm 1.9$ per $160 \mu \mathrm{m})$ and hair cells $(1.5 \pm 0.3$ per 160 $\mu \mathrm{m}$ ) in the apical turn (Figure $2 \mathrm{H}$ ), with EdU-labeled supporting cells also present in the middle turn (Figure 2I) but not the basal turn (Figure 2J). On average, there was a 9.2-fold increase in the total number of EdU-labeled cells in damaged, Sox $2^{\text {haplo }}$ cochleae compared with total numbers in the damaged-only cochleae (Figure 2K and Supplemental Table 1). These results indicate that Sox $2^{\text {haplo }}$ increases the degree and expands the domain of damage-induced proliferation along the length of the cochlea.

Hair cell regeneration in the neonatal mammalian cochlea can also occur without an antecedent mitotic event $(43,45)$, through the direct transition of supporting cells into hair cells. We directly assessed the degree of new hair cell formation through immunostaining of damaged cochleae for the transcription factor Atoh1 (Figure 3, A and B, and Supplemental Figure 3A), which is upregulated in nonmitotic hair cell precursors in the regenerating mouse vestibular system $(46,47)$. Atoh1 is highly expressed in nascent hair cells in the embryonic cochlea and is rapidly downregulated postnatally $(48,49)$. In agreement with previous reports, we detected Atoh1 protein expression in hair cells in the E18.5 cochlea and its reduction in a base-to-apex direction between PO and $\mathrm{P} 3$, with no expression detected in hair cells at P4 or in supporting cells at any of these ages (Figure 3C and Supplemental Figure 2, A-L). We examined the P1 Atoh $1^{\text {GFP/+ }}$ cochlea (50) and found a similar apical-basal gradient of GFP expression in hair cells and none in supporting cells (Supplemental Figure 2, M-O). Two days after DT-induced hair cell loss on P1, we detected Atoh1, Sox2 double-positive supporting cells in the apical and middle turns in damaged-only (Pou4f3 $3^{\text {DTR/+}}$ ) mouse cochleae (Figure 3, D and E, and Supplemental Figure 3, B-D). To ascertain whether the Atoh1 ${ }^{+}$ cells were supporting cells acquiring a hair cell phenotype, we examined P3 cochlea and found that Atoh $1^{+}$Sox $2^{+}$supporting cells also expressed Gfi1, another hair cell transcription factor normally absent in supporting cells (Figure 3D and Supplemental Figure 3, $\mathrm{F}$ and I). The following day (P4), we found that many Atoh1 $1^{+}$Sox $2^{+}$ cells also expressed myosin $7 \mathrm{a}$ in the apical and middle turns and 
A

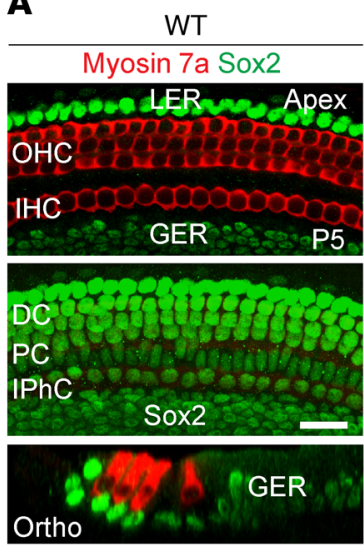

B

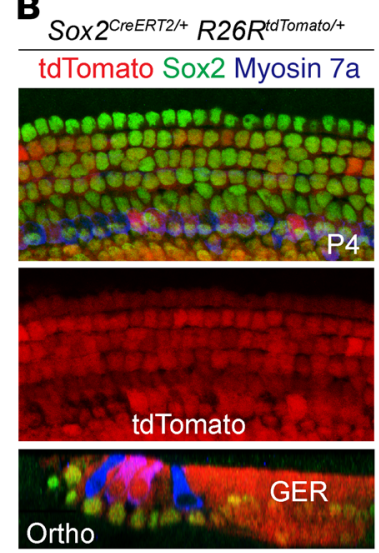

C

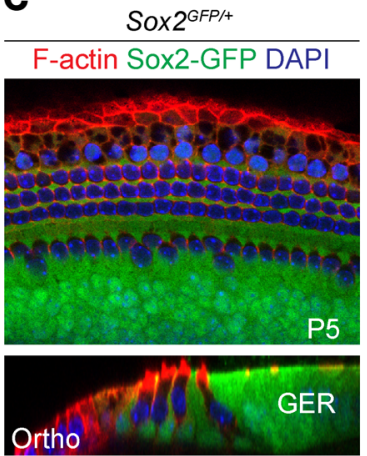

Figure 1. Sox2 haploinsufficiency results in continued proliferation and formation of supernumerary hair cells in the neonatal cochlea.

(A) Immunostaining of P5 WT cochlea shows Sox2 expression in Hensen's cells, Deiters' cells, pillar cells, and the lateral portion of the greater epithelial ridge. (B) Whole-mount preparation of cochlea from P4 Sox $2^{\text {CreERT2/+ }} R 26 R^{\text {tdTomato/t }}$

mice given tamoxifen on $\mathrm{P} 2$, showing tdTomato expression in supporting cells and some hair cells. (C) GFP+ supporting cells in the P5 Sox $2^{\mathrm{CFP} /+}$ cochlea. (D) Schematic of EdU administration to Sox $2^{\text {CreERT2/+ }}$ mice, Sox $2^{\mathrm{CFP} /+}$ mice, and WT littermates (once daily, P2-P4). haplo, haploinsufficient. (E) QPCR showed a significant reduction of Sox2 expression in Sox $2^{\text {CreERT2/+ }}$ cochleae compared with expression in WT littermates. (F) Confocal images show no EdU+ hair cells or supporting cells in the P5 WT cochlea. EdU labeling was seen in cells in the lesser epithelial ridge and greater epithelial ridge. (C) Sox $2^{\text {CreERT2/+ }}$ cochlea contained occasional extranumerary hair cells adjacent to inner hair cells (arrowheads). Extranumerary hair cells were noted in all cochlear turns of Sox2 $2^{\text {CreERT2/+ }}$ mice. Image shows EdU+ supporting cells (chevrons) in the apical turn. No EdU+ hair cells were noted. (H) Quantification of extranumerary hair cells in WT, Sox $2^{\text {CreERT2/+ }}$, and Sox $2^{\mathrm{CFP} /+}$ cochleae. (I) Quantification of EdU+ cells in WT, Sox $2^{\text {CreERT2/+ }}$, and Sox $2^{\text {CFP/+ }}$ cochleae. (J) P28 Sox2 $2^{\text {CreERT2/+ }}$ mice had normal ABR thresholds comparable to those of their WT littermates. DC, Deiters' cell; GER, greater epithelial ridge; HC, hair cell; IHC, inner hair cell; IP, inner pillar cell; IPhC, inner phalangeal cell; LER, lesser epithelial ridge; $\mathrm{OHC}$, outer hair cell; OP, outer pillar cell; Ortho, orthogonal view; PC, pillar cell; SC, supporting cell. Data represent the mean \pm SD. ${ }^{*} P<0.05$ and ${ }^{* *} P<0.01$, by 2 -tailed Student's $t$ test. $n=3-8$. Scale bar: $20 \mu \mathrm{m}$
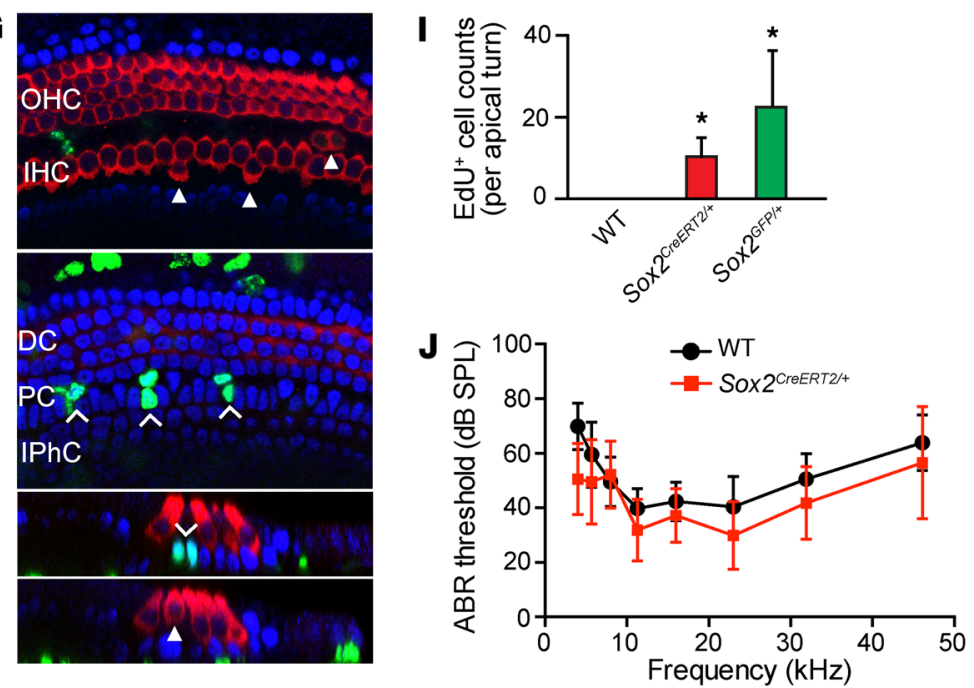

rarely in the base $(9.7 \pm 0.6,5.0 \pm 1.0$, and $0.3 \pm 0.6$, respectively) (Figure 3G, Supplemental Figure 3, G and J, and Supplemental Table 2). By P4, when cochlear hair cells normally lack Sox2 expression (Figure 3F), we found that Atoh $1^{+}$myosin $7 \mathrm{a}^{+}$cells also expressed Sox 2 and Gfi1 (Figure 3G), suggesting that they were supporting cells transitioning into nascent hair cells (Figure $3 \mathrm{~B}$ ). Given these findings, we hereafter define Atoh $1^{+}$Sox $2^{+}$cells as transitional cells. Thus, unlike the proliferative cells that were limited to the apical turn of the damaged cochlea, Atoh1 ${ }^{+}$transitional cells were found in all 3 cochlear turns (Figure 3I, and Supplemental Figure 3, G and J). In comparison with the damaged-only cochlea (P4), the damaged, Sox $2^{\text {haplo }}$ cochlea contained a significantly larger number of transitional cells in all turns (Figure 3, $\mathrm{H}$ and I, and Supplemental Figure 3, H and K). Collectively, these 
A

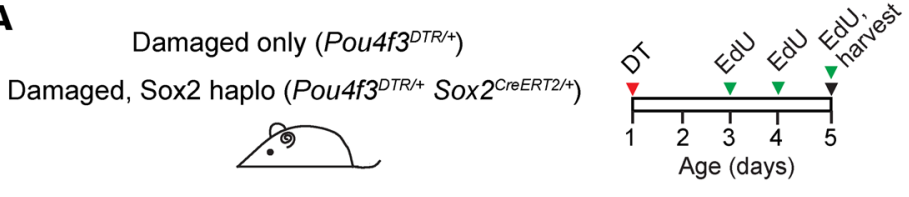

Myosin 7a EdU Sox2
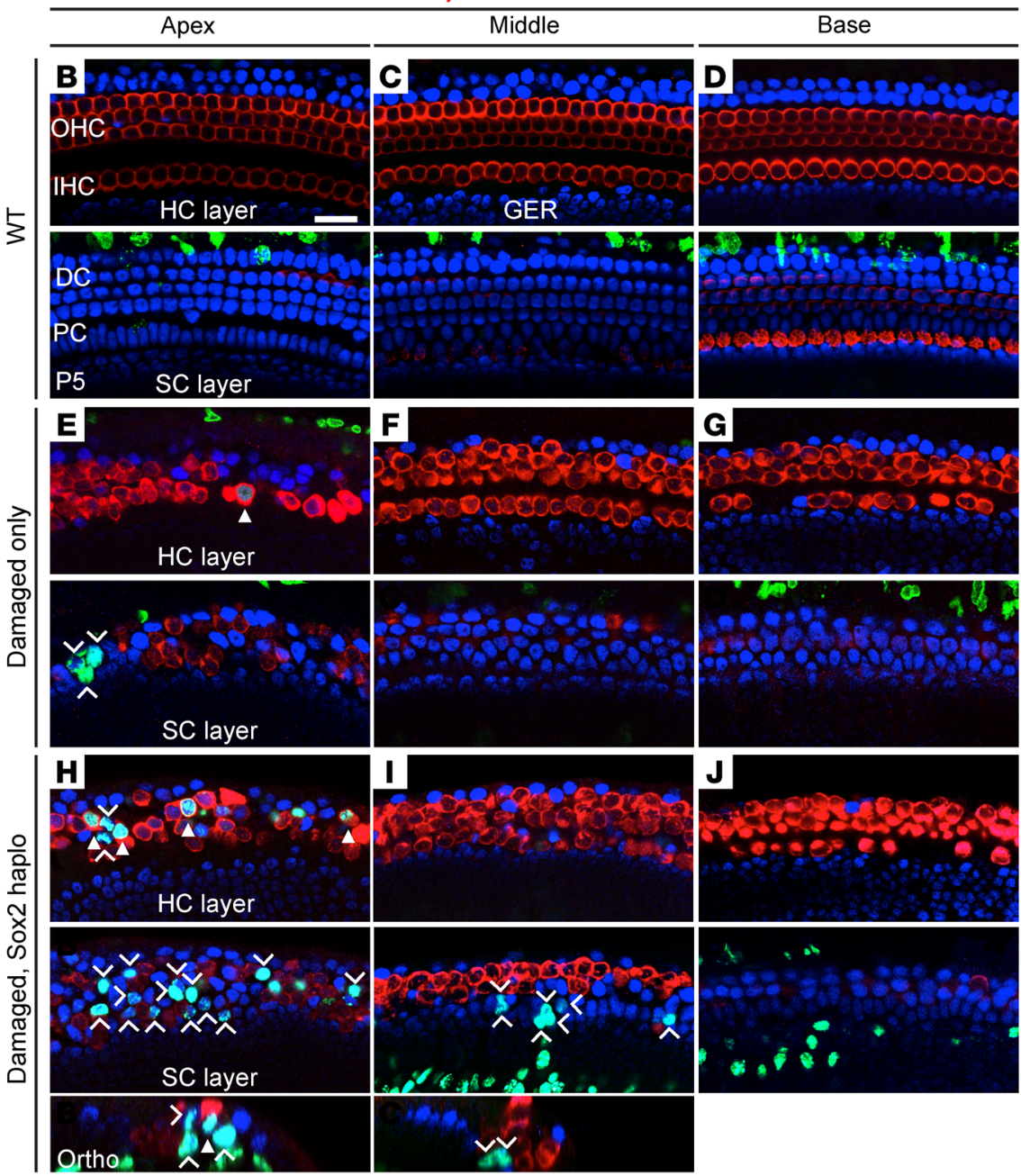

$\mathbf{K}$

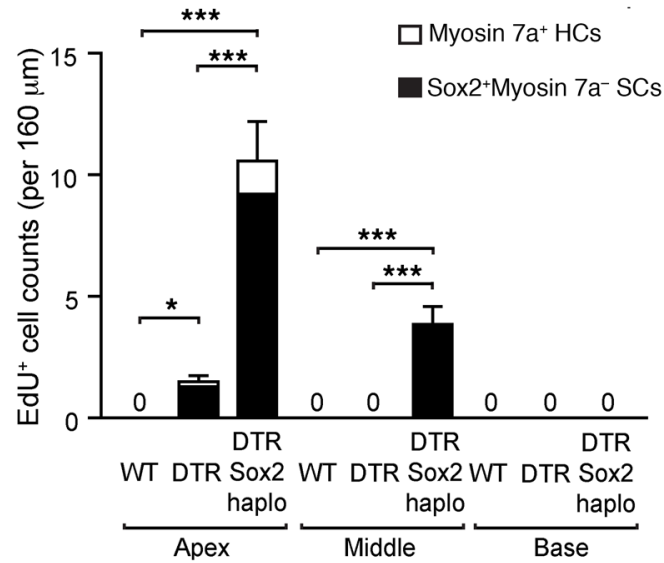

Figure 2. Reduced Sox2 levels enhance and expand the domain of proliferation in the damaged neonatal mouse cochlea. (A) Schematic of hair cell ablation in neonatal cochlea. Briefly, Pou $4 f 3^{D T R /+}$ and Pou $4 \mathrm{f}^{\text {DTR/+ }}$ Sox $2^{\text {CreERT2/+ }}$ mice were injected with DT on P1 to induce hair cell loss, followed by administration of EdU (P3-P5), and cochleae were examined on P5. (B-D) No EdU' hair cells or supporting cells were found in any of the 3 WT cochlear turns. (E-C) In the Pou $4 f 3^{\mathrm{DTR} /+}$ cochlea, after DT-induced hair cell damage, EdU+myosin $7 a^{+}$hair cells (arrowhead) and some EdU ${ }^{+} \mathrm{Sox2}^{+}$supporting cells (chevrons) were observed in the apical turn, but not in the basal or middle turns. (H-J) In Pou4f3 $3^{\text {DTR/+ }}$ Sox $2^{\text {CreERT2/+ }}$ cochlea, there was robust EdU labeling of both myosin $7 \mathrm{a}^{+}$hair cells (arrowheads) and Sox $2^{+}$supporting cells (chevrons) in the apical turn. EdU $+\mathrm{Sox2}^{+}$supporting cells were also found in the middle turn. (K) Quantification of EdU'myosin $7 a^{+}$hair cells and myosin $7 a^{-}$Sox $2^{+}$ supporting cells per cochlear turn. Data represent the mean \pm SD. ${ }^{*} P<0.05$ and ${ }^{* *} P<0.001$, by 1 -way ANOVA with Holm-Sidak multiple comparisons test. $n=5-6$. Scale bar: $20 \mu \mathrm{m}$.

results suggest that Sox ${ }^{\text {haplo }}$ enhances transitional cell formation in the damaged neonatal cochlea.

Sox $2^{\text {haplo }}$ promotes the survival of regenerated hair cells. A previous study showed that regenerated hair cells in the neonatal mouse cochlea undergo subsequent cell death (51). To determine whether Sox $2^{\text {haplo }}$ promotes the survival of regenerated hair cells, we compared the damaged, Sox $2^{\text {haplo }}$ cochlea with the damaged-only cochlea on P21 (after treatment with DT on P1 and with EdU from P3 to P5) (Supplemental Figure 4A). As expected, we detected no EdU incorporation in the WT cochlea (Supplemental Figure 4B). We found that the damaged-only cochlea contained few hair cells among disorganized supporting cells in the apical turn, with occasional EdU labeling of each cell type (Supplemental Figure 4, C and F). In contrast, the Sox $2^{\text {haplo }}$, damaged cochleae had significantly more $\mathrm{EdU}^{+}$hair cells and supporting cells in the apical turn than did the damaged-only cochleae (Supplemental Figure 4, D and F). Early after damage (P5, P7, and P10), we found a significantly greater number of hair cells in most turns of the Sox $2^{\text {haplo }}$, damaged cochleae than in the damaged-only cochleae, even though there were few EdU-labeled cells in the middle turn and none in the basal turn on P5 (Supplemental Tables 1 and 3). By P21, both the damaged-only and Sox $2^{\text {haplo }}$, damaged cochleae had a gradual loss of hair cells and EdU-labeled cells (Supplemental Figure 4, E and F, and Supplemental Table 3), with hair cells remaining only in the apical turn. 
A

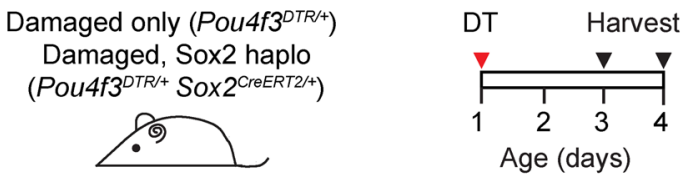

B

Supporting cell Transitional cell? HC-like
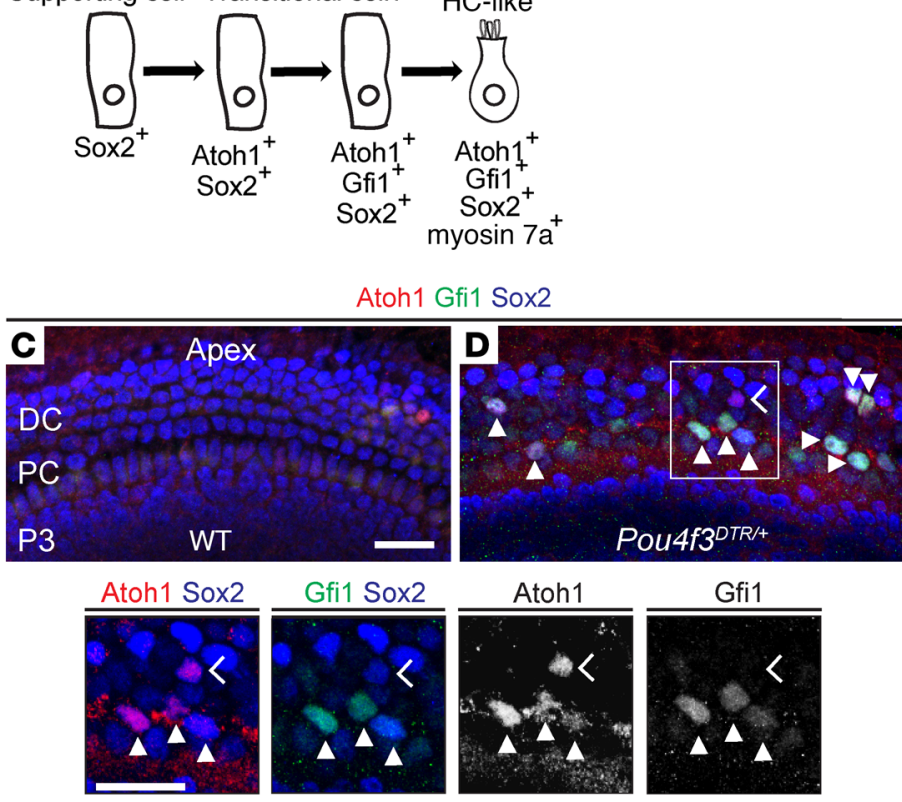

Atoh1 Gfi1 Sox2 Myosin 7a
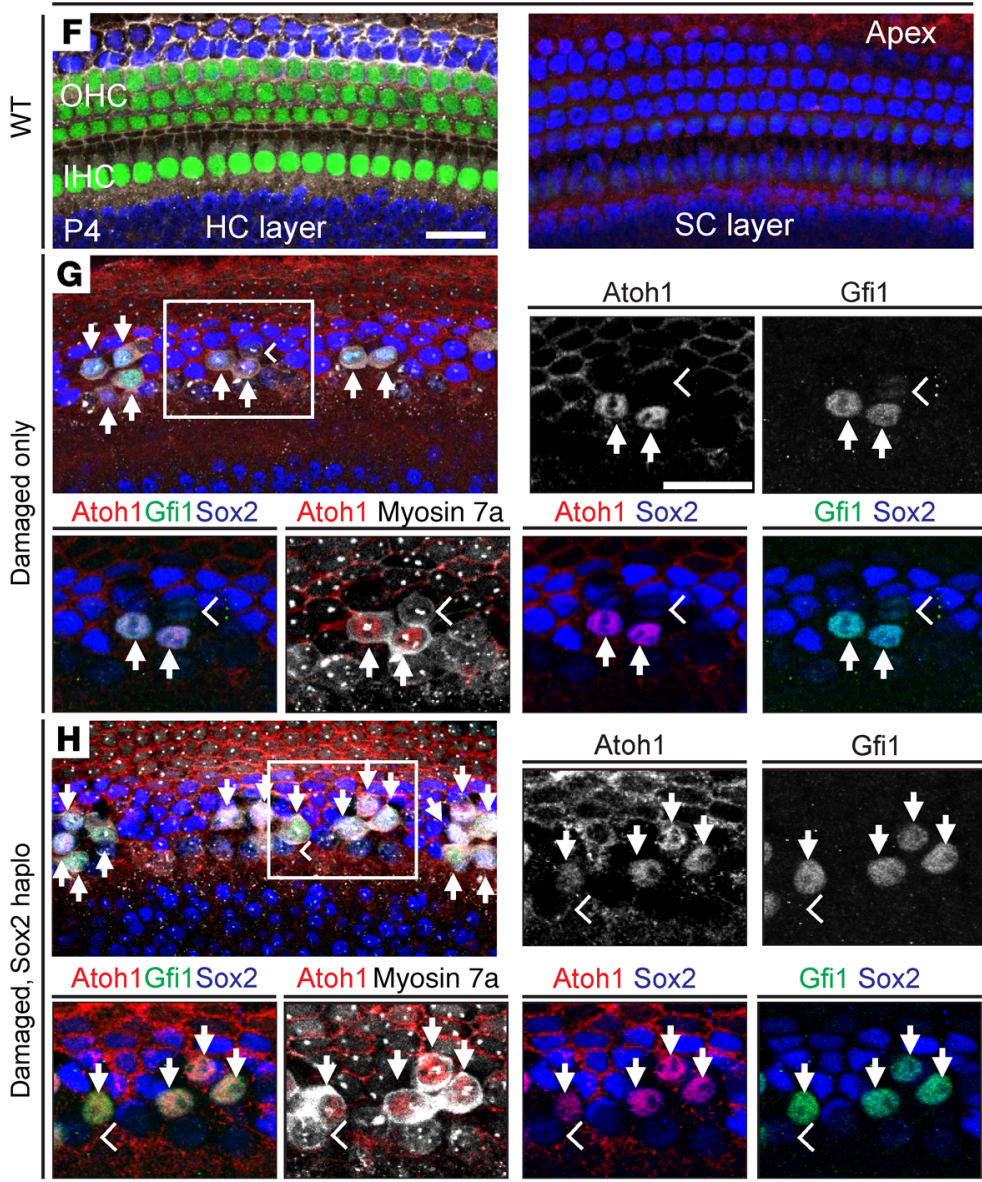
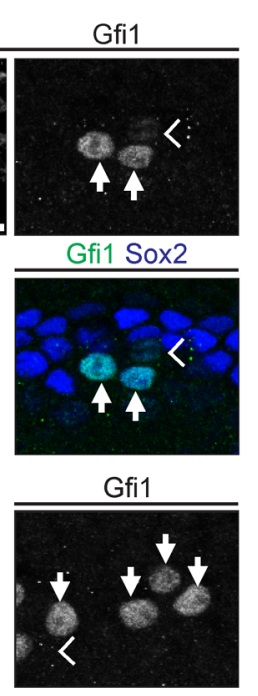

Gfi1 Sox2

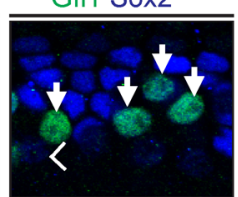

Figure 3. Sox2 reduction enhances transitional cell formation in the damaged neonatal mouse cochlea. (A) Schematic of hair cell ablation. P1 Pou $4 f 3^{\text {DTR/+ }}$ and Pou $4 f 3^{\text {DTR/+ }}$ Sox $2^{\text {CreERT2/+ }}$ pups were injected with $\mathrm{DT}$, and cochleae were examined on $\mathrm{P} 3$ and P4. (B) Cartoon depicts supporting cells forming transitional cells during regeneration. (C) Confocal images of P3 WT cochlea show no Atoh1 or Gfi1 expression in supporting cells. (D) After hair cell damage in the Pou $4 \mathrm{f}^{\mathrm{DTR} /+}$ cochlea, some Sox $2^{+}$supporting cells expressed Atoh1 (chevrons) and Gfi1 (arrowheads), both early hair cell markers. All Gfi1 ${ }^{+}$Sox2 ${ }^{+}$supporting cells expressed Atoh1, but some Atoh $1^{+}{ }^{-}$ox $2^{+}$supporting cells did not express Gfi1. (E) Quantification of transitional cells (Atoh1 $1^{+}$Sox2 ${ }^{+}$and Atoh $1^{+}$Sox2 $2^{+} \mathrm{Cfi1}^{+}$) from WT and Pou $4 \mathrm{f}^{\mathrm{DTR} /+}$ cochleae. (F) P4 WT cochlea showed Gfi1 expression limited to hair cells and no Atoh1 or Gfi1 expression in Sox $2^{+}$supporting cells. Atoh1 was absent in hair cells. (G) After DT-induced hair cell loss in Pou $4 \mathrm{f}^{\text {DTR/+ }}$ cochlea, Gfi1 was downregulated in the remaining myosin $7 a^{+}$hair cells. Many transitional cells (arrows) (Atoh1+5ox2+myosin $7 \mathrm{a}^{+} \mathrm{C}$ fi1 $1^{+}$) were detected in all 3 cochlear turns. Like the P3 Pou $4 \mathrm{f3}^{\mathrm{DTR} /+}$ cochlea, all transitional cells expressed Atoh1 and Sox2. In contrast to the P3 Pou4f3 $3^{\text {DTR/+ }}$ cochlea, most transitional cells expressed myosin 7a by P4. Myosin7a+ cells with no expression of Sox2, Atoh1, or Gfi1 (chevron) likely represent surviving hair cells (G and $\mathbf{H})$. (H) In the P4 Pou4f3 ${ }^{\text {DTR/+ Sox }} 2^{\text {CreERT2/+ }}$ cochlea, there were noticeably more transitional cells (arrows). (I) Quantification of transitional cells (Atoh1 ${ }^{+}$Sox2 $2^{+}$myosin $7 a^{+} \mathrm{Cfi}^{+}$and Atoh $1^{+}$Sox2 ${ }^{+} \mathrm{Cfi} 1^{+}$) in WT, Pou $4 \mathrm{f}^{\text {DTR/+}}$, and Pou $4 \mathrm{f}^{\text {DTR/+ }}$ Sox $2^{\text {CreERT2/+ }}$ cochleae. Hair cell ablation led to a significantly greater number of transitional cells in each cochlear turn compared with that seen in controls. There were significantly more transitional cells detected in each turn of damaged, Sox $2^{\text {haplo }}$ cochleae than in the damagedonly cochleae. The SD of transitional cell counts in the basal turn of damaged, Sox $2^{\text {haplo }}$ cochleae is zero. Data represent the mean $\pm \mathrm{SD}$. ${ }^{*} P<0.05$, ${ }^{*} P<0.01$, and ${ }^{* *} P<0.001$, by 1 -way ANOVA with Holm-Sidak multiple comparisons test. $n=3$. Scale bars: $20 \mu \mathrm{m}$.

\section{$\mathbf{E}$}
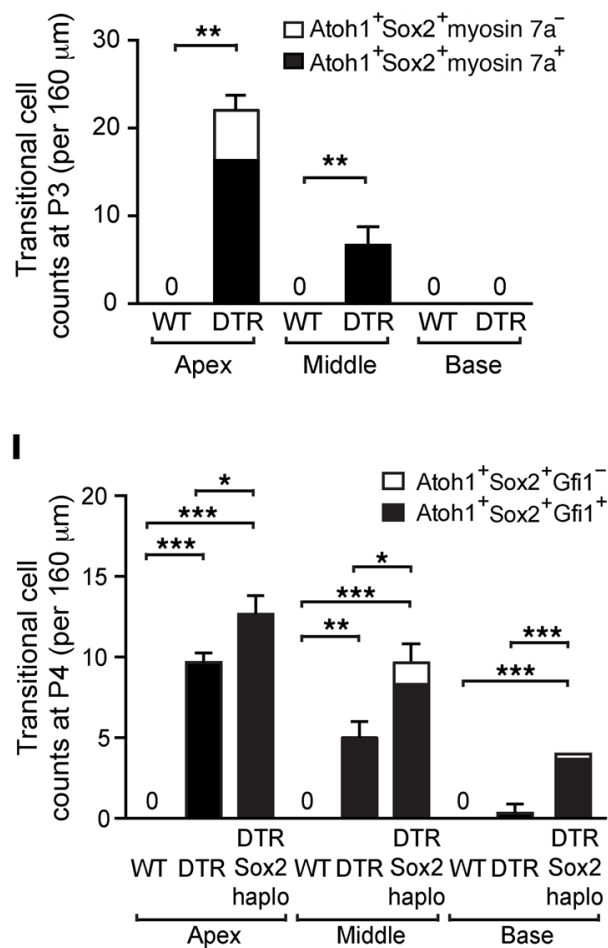
There were, however, still significantly more hair cells in the Sox $2^{\text {haplo }}$, damaged cochleae than in the damaged-only cochleae. After DT administration, both Pou $43^{3^{D T R /+}}$ and Pou $4 \mathrm{f}^{\text {DTR/+ }}$ So $2^{\text {CreERT2/+ }}$ animals showed markedly elevated auditory thresholds (Supplemental Figure 4G). Taken together, these results indicate that Sox $2{ }^{\text {haplo }}$ modestly promotes the survival of regenerated hair cells in the damaged cochlea, but the extent of survival is insufficient to establish auditory function.

So $2^{\text {haplo }}$ enhances $\beta$-catenin-induced proliferation in the damaged neonatal cochlea. $\beta$-Catenin is the central mediator of the canonical Wnt signaling pathway, with exon 3 encoding a domain to be phosphorylated by glycogen synthase $3 \beta$. Phosphorylated $\beta$-catenin is degraded by the destruction complex, rendering the pathway inactive (52). In heterozygotes carrying $\beta$-catenin lacking exon 3 , $\beta$-catenin is stabilized, leading to aberrant activation of Wnt signaling, and thus acts as a dominant mutation (53). Previous studies show that $\beta$-catenin stabilization in the neonatal cochlea causes proliferation and ectopic hair cell formation $(54,55)$. To further explore its role after damage, we first used 2 Cre-LoxP model systems to enhance Wnt signaling by stabilizing $\beta$-catenin in neonatal supporting cells (Figure 4, A and B). In Fgfr3-iCre mice given tamoxifen at P2, we detected Cre activity in approximately $86.9 \%$ of supporting cells in the P4 cochlea (using Ai14 tdTomato reporter mice; data not shown). To determine whether $\beta$-catenin stabilization enhances mitotic regeneration, we examined Pou4f3 $3^{\text {DTR/+ }}$ Fgfr3-iCre Ctnnb1 ${ }^{f(\text { lex3)/+ }}$ (referred to hereafter as damaged, $\beta$-catenin ${ }^{\mathrm{GOF}}$ ) cochleae and compared them with damagedonly $\left(\right.$ Pou $43^{\text {DTR/+}}{ }^{-}$cochleae. We observed significantly more $(\sim 3.5-$ fold) EdU-labeled supporting cells and hair cells in the damaged, $\beta$-catenin ${ }^{\mathrm{GOF}}$ cochleae, a finding that was limited to the apical turn (Figure 4, C-E, and I, and Supplemental Table 1). This suggests that $\beta$-catenin ${ }^{\mathrm{GOF}}$ increases mitotic regeneration without expanding its domain to the middle or basal turns.

We next probed the effects of Sox $2^{\text {haplo }}$ on $\beta$-catenininduced mitotic regeneration by examining damaged, Sox $2^{\text {haplo, }}$, $\beta$-catenin ${ }^{\mathrm{GOF}}\left(\right.$ Pou $_{4} \mathrm{f}^{\mathrm{DTR} /+}$ Sox $2^{\text {CreERT2/+}}$ Ctnnb1 $\left.^{f(\text { (ex3)/+}}\right)$ cochleae. We found many EdU $\mathrm{E}^{+}$supporting cells and hair cells in both the apical and middle turns and also observed EdU ${ }^{+}$supporting cells in the basal turn (Figure 4, F-I, and Supplemental Table 1). In comparison with the damaged, $\beta$-catenin ${ }^{\mathrm{GOF}}$ cochlea, we observed an additional increase in the number of $\mathrm{EdU}^{+}$supporting cells and hair cells in the apical and middle turns (Figure 4I and Supplemental Table 1). Relative to the damaged, Sox $2^{\text {haplo }}$ cochlea, the damaged, Sox $2^{\text {haplo }}, \beta$-catenin ${ }^{\mathrm{GOF}}$ cochlea contained more $\mathrm{EdU}^{+}$supporting cells and hair cells in the apical turn only, while the cells in the middle and basal turns were not significantly different (Supplemental Table 1). These results indicate that both $\beta$-catenin ${ }^{\mathrm{GOF}}$ and Sox $2^{\text {haplo }}$ enhanced mitotic regeneration in the apical turn in the damaged cochlea. While Sox $2^{\text {haplo }}$ extended the domain of mitotic regeneration into the middle and basal turns of the damaged cochlea, $\beta$-catenin ${ }^{\mathrm{GOF}}$ failed to induce proliferation in this region, thus its mitogenic effect is spatially restricted to the apex.

So $2^{\text {haplo }}$ acts as a permissive signal for $\beta$-catenin-responsive transitional cell formation in the damaged neonatal cochlea. To assess whether $\beta$-catenin ${ }^{\mathrm{GOF}}$ also enhances transitional cell formation, we stained for Atoh1 and Gfi1 in the damaged, $\beta$-catenin ${ }^{\mathrm{GOF}}$ cochlea (P4) and found transitional cells (Atoh $1^{+} \mathrm{Gfi} 1^{+}$Sox $2^{+}$) in the apical and middle turns (Figure 5, A, B, and D). However, we detected no transitional cells in the base (Figure 5F). Compared with damagedonly cochlea without stabilized $\beta$-catenin, fewer transitional cells expressed myosin 7a, although there was no significant change in the total number (Figure $5 \mathrm{H}$ and Supplemental Table 2 ), suggesting that $\beta$-catenin ${ }^{\mathrm{GOF}}$ alone does not enhance transitional cell formation.

We next assessed whether Sox $2^{\text {haplo }}$ affects $\beta$-catenin-induced transitional cell formation and examined damaged, Sox $2^{\text {haplo, }}$ $\beta$-catenin ${ }^{\mathrm{GOF}}$ cochleae. We detected a robust and significant increase in the number of transitional cells in all 3 cochlear turns compared with cell numbers in damaged, $\beta$-catenin ${ }^{\mathrm{GOF}}$ or damaged-only (Pou4f3 $3^{\text {DTR/+}}$ ) cochleae (Figure 5, C, E, G, H, and Supplemental Table 2). Furthermore, to distinguish the effects of Sox $2^{\text {haplo }}$ from those of $\beta$-catenin ${ }^{\mathrm{GOF}}$, we compared the effects and found that the number of transitional cells was significantly higher in damaged, Sox $2^{\text {haplo }}, \beta$-catenin ${ }^{\mathrm{GOF}}$ cochleae than in damaged, Sox ${ }^{\text {haplo }}$ cochleae without stabilized $\beta$-catenin (Supplemental Table 2). Taken together, these data suggest that both Sox $2^{\text {haplo }}$ and damage act as permissive signals for $\beta$-catenin-induced transitional cell formation in the neonatal cochlea.

So $2^{\text {haplo }}$ primes the neonatal cochlea for $\beta$-catenin-induced proliferation. We next probed the relationship between Sox $2^{\text {haplo }}$ and $\beta$-catenin ${ }^{\mathrm{GOF}}$ by examining the undamaged cochlea. After administering tamoxifen on $\mathrm{P} 2$ to $\beta$-catenin ${ }^{\mathrm{GOF}}$ (Fgfr3-iCre Ctnnb1 ${ }^{(e \times 3) /+}$ ) mice, we did not detect any EdU-labeled supporting cells or ectopic hair cell formation in the P5 cochlea (Figure 6, A and B, and Supplemental Figure 5, A-C). However, after the same tamoxifen and EdU regimen, we found many EdU ${ }^{+}$supporting cells (but not hair cells) in the pillar cell region in the Sox $2^{\text {haplo }}, \beta$-catenin ${ }^{\text {GOF }}$ (Sox $2^{\text {CreERT2/+ }}$ Ctnnb1 ${ }^{f(\text { f(ex3)/+})}$ cochleae (Figure 6, A, C, and G, and Supplemental Figure 5, A, D, and E). To test whether cochlear supporting cells become $\beta$-catenin responsive upon acute downregulation of Sox 2 , we next concurrently stabilized $\beta$-catenin and partially deleted Sox2 by using Fgfr3-iCre Sox $2^{f l /+}$ Ctnnb1 $1^{f(e x 3) /+}$ (conditional Sox 2 haplo $\beta$-catenin ${ }^{\mathrm{GOF}}$ ) mice (Figure $6 \mathrm{D}$ and Supplemental Figure 5F) (32). As expected, no EdU ${ }^{+}$cells were detected in cochleae from $\beta$-catenin ${ }^{\mathrm{GOF}}$ (Fgfr3-iCre Ctnnb1 $^{\text {fl(ex3)/+})}$ mice given tamoxifen on P1 and EdU from P3 to P5 (Figure 6E, and Supplemental Figure 5, G, and H). This contrasts with the conditional Sox $2^{\text {haplo }}, \beta$-catenin ${ }^{\mathrm{GOF}}$ (Fgfr3-iCre Sox $2^{f l++}$ Ctnnb1 $^{f(\text { lex3)/+}}$ ) cochleae, in which we observed many EdU ${ }^{+}$supporting cells in the apical and middle turns (Figure 6, F and G, and Supplemental Figure 5, I and J).

To directly assess the effects of Sox ${ }^{\text {haplo }}$ on Wnt signaling, we immunostained for the Wnt target Lef1 (56). In the P5 WT cochlea, we found that Lef1 expression was restricted to below the basilar membrane, where Wnt-responsive, tympanic border cells reside, as previously described (57), and we detected no expression in the sensory epithelium (Supplemental Figure 6, A-C). Similarly, we failed to detect Lef1 in the sensory epithelium from $\beta$-catenin ${ }^{\mathrm{GOF}}$ or Sox $2^{\text {haplo }}$ mice (Supplemental Figure 6, D-I). In the organ of Corti from Sox $2^{\text {haplo }}, \beta$-catenin ${ }^{\text {GOF }}$ cochlea, we found many Lef1 ${ }^{+}$ supporting cells in the apical turn, some of which were arranged as foci within the pillar cell region (Supplemental Figure 6, J-L). Thus Sox $2^{\text {haplo }}$ acts as a permissive signal for $\beta$-catenin-induced Wnt activation in the undamaged, neonatal cochlea. 
A

Damaged, $\beta$-catenin ${ }^{\mathrm{GOF}}$ (Pou4f3 ${ }^{D T R /+}$ Fgfr3-iCre Ctnnb1 $1^{\text {fl(ex3)/+}}$ ) Damaged, Sox 2 haplo, $\beta$-catenin ${ }^{\mathrm{GOF}}$ (Pou4f3 ${ }^{\text {DTR/+ }}$ Sox $2^{\text {CreERT2/+ }}$ Ctnnb1 $1^{f(\mathrm{e} \times 3) /+}$ )
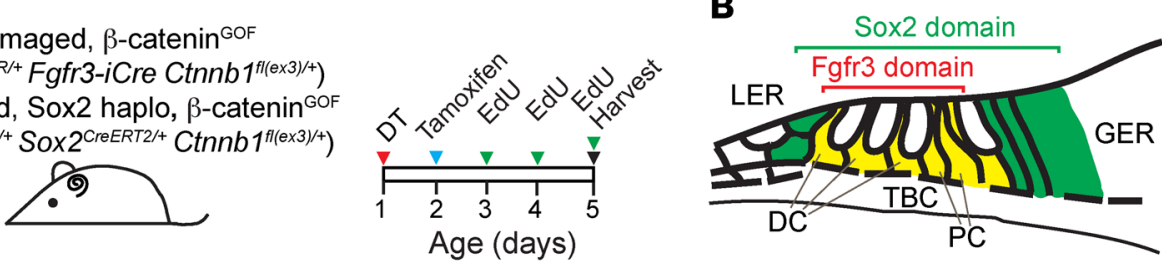

Myosin 7a EdU Sox2

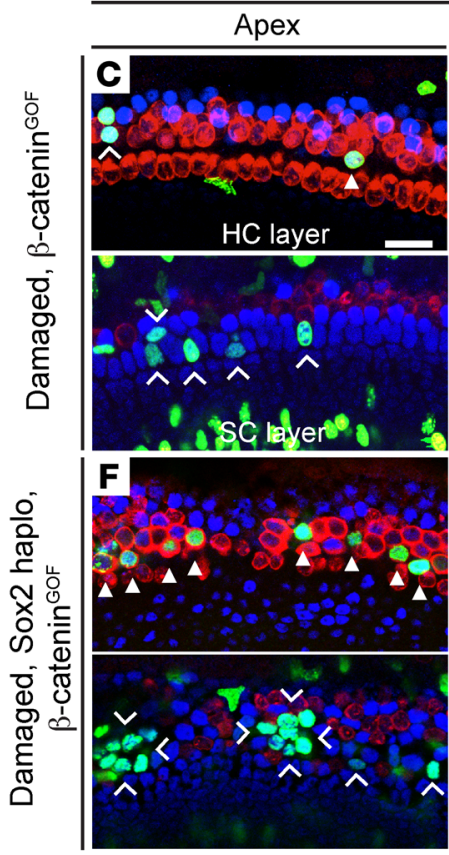

Middle
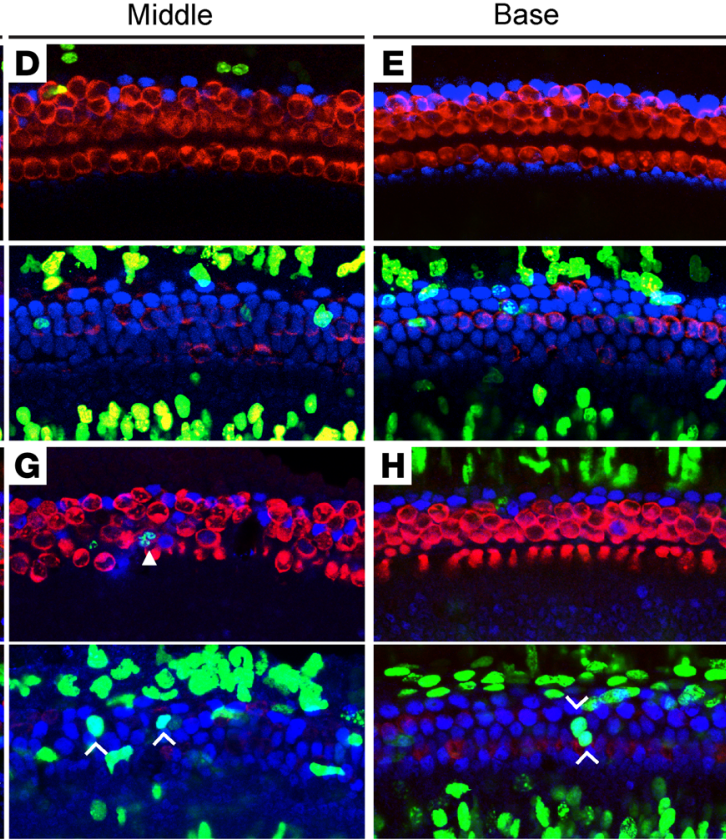

I

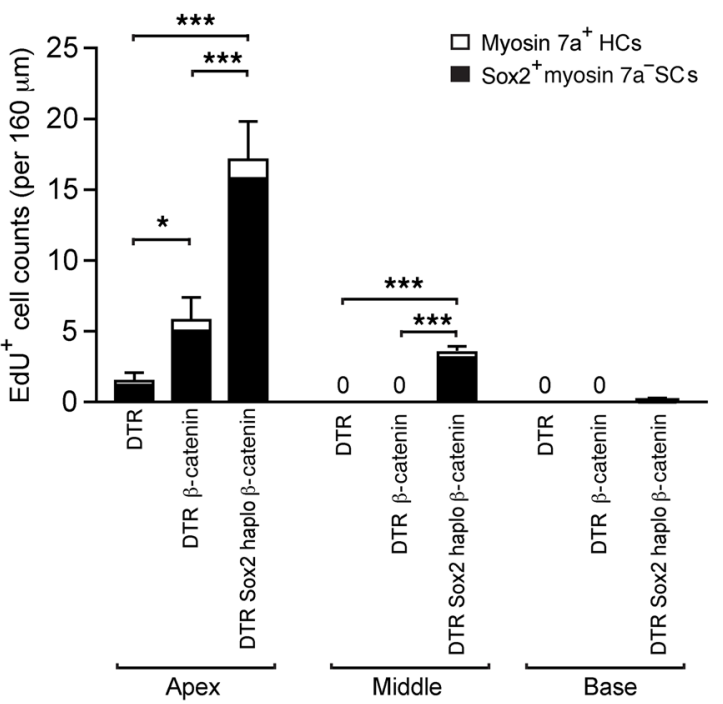

Figure 4. $\beta$-Catenin stabilization and Sox 2 haploinsufficiency coordinate to increase mitotic regeneration in the damaged neonatal mouse cochlea. (A)

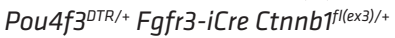
and Pou $4 \mathrm{f}^{\mathrm{OTR} /+}$ Sox $2^{\mathrm{Cr} \text { reRT2/+ }}$ Ctnnb ff(ex3)/++ pups were injected with DT on P1, tamoxifen on P2, and EdU daily (P3-P5), and cochleae were collected on P5. (B) Schematic depicting the domains of $\mathrm{Fgfr} 3$ and Sox2 expression in the neonatal mouse cochlea. (C-E) Confocal images of cochleae from $\mathrm{P} 5$ Pou4f3 $3^{\text {DTR/+}}$ Fgfr3-iCre Ctnnb $7^{f(\text { lex } 3) /+}$ mice showing EdU+myosin $7 a^{+}$hair cells (arrowhead) and EdU ${ }^{+}$Sox $2^{+}$ supporting cells (chevrons) in the apical turn, but not in the middle or basal turn. Note that many EdU'Sox2- cells resided outside the sensory epithelium. (F-H) In Pou $43^{\text {DTR/+ }}$ Sox $^{\text {CreERT2/+ }}$ Ctnnb $7^{f(\operatorname{lex} 3) /+}$ cochlea, there was a robust increase in the number of EdU ${ }^{+}$myosin $7 a^{+}$ hair cells (arrowheads) and Sox $2^{+}$ supporting cells (chevrons) in the apical turn. As with Pou4f $3^{\text {DTR/+ }}$ Sox $2^{\text {CreERT2/+ }}$ cochlea, EdU++ supporting cells were noted in the middle turns and occasionally in the basal turns. Many EdU+Sox2- cells outside the sensory epithelium were also noted. (I) Quantification of EdU' myosin $7 a^{+}$hair cells and $\mathrm{EdU}^{+} \mathrm{Sox2}{ }^{+}$supporting cells in Pou4f3 $3^{\text {DTR/+ }}$, Pou4f3 $3^{\text {DTR/+ }}$ Fgfr3-iCre Ctnnbifl(ex3)/+ , and Pou $4 \mathrm{f3}^{\mathrm{DTR} /+}$ Sox2 $2^{\text {CreERT2/+ }}$ Ctnnb $7^{f(\text { (ex3)/++ }}$ cochleae. Data represent the mean \pm SD. ${ }^{*} P<0.05$ and ${ }^{* *} P<0.001$, by 1-way ANOVA with Holm-Sidak multiple comparisons test. $n=3-5$ Scale bar: $20 \mu \mathrm{m}$.
We next immunostained for Atoh1 and Gfi1 to detect transitional cells in the undamaged, Sox $2^{\text {haplo }}, \beta$-catenin ${ }^{\mathrm{GOF}}$ cochlea

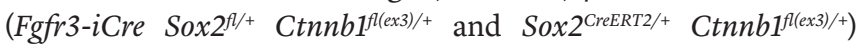
(Figure $6 \mathrm{H}$ and Supplemental Figure 5K). We detected no Atoh1 expression and found that Gfil expression was confined to hair cells in cochleae subjected to $\beta$-catenin stabilization only (Fgfr3-iCre Ctnnb1 ${ }^{f(e x 3) /+}$ ) (Figure 6I and Supplemental Figure 5, L and $\mathrm{M})$. Similarly, no transitional cells were found in the undamaged, Sox $2^{\text {haplo }}, \beta$-catenin ${ }^{\mathrm{GOF}}$ cochleae from either model (Fig- ure 6, J and K, and Supplemental Figure 5, N-Q). These data indicate that Sox 2 haplo acts as a permissive signal for $\beta$-catenin-induced proliferation in the undamaged, neonatal cochlea. Moreover, we found that, in the absence of damage and Sox $2^{\text {haplo }}, \beta$-catenin ${ }^{\mathrm{GOF}}$ does not induce transitional cell formation.

Sox $2^{\text {haplo }}$ and $\beta$-catenin ${ }^{G O F}$ do not promote proliferation or hair cell formation in the mature cochlea. To test whether Sox $2^{\text {haplo }}$ and $\beta$-catenin ${ }^{\mathrm{GOF}}$ can induce regeneration in the damaged, mature cochlea, we used 2 models of hair cell ablation: aminoglycoside 
A

Damaged, $\beta$-catenin ${ }^{\mathrm{GOF}}$

(Pou4f3 ${ }^{\text {DTR/+ }}$ Fgfr3-iCre Ctnnb1 $1^{\text {flex3)/+ }}$ )

Damaged, Sox2 haplo, $\beta$-catenin ${ }^{\mathrm{GO}}$

(Pou4f3 ${ }^{\text {DTR/+ }}$ Sox2 $2^{\text {CreERT2/+ }}$ Ctnnb $1^{1 /((\mathrm{ex} 3) /+}$ )

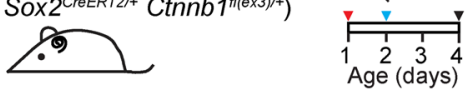

Atoh1 Gfi1 Sox2 Myosin 7a
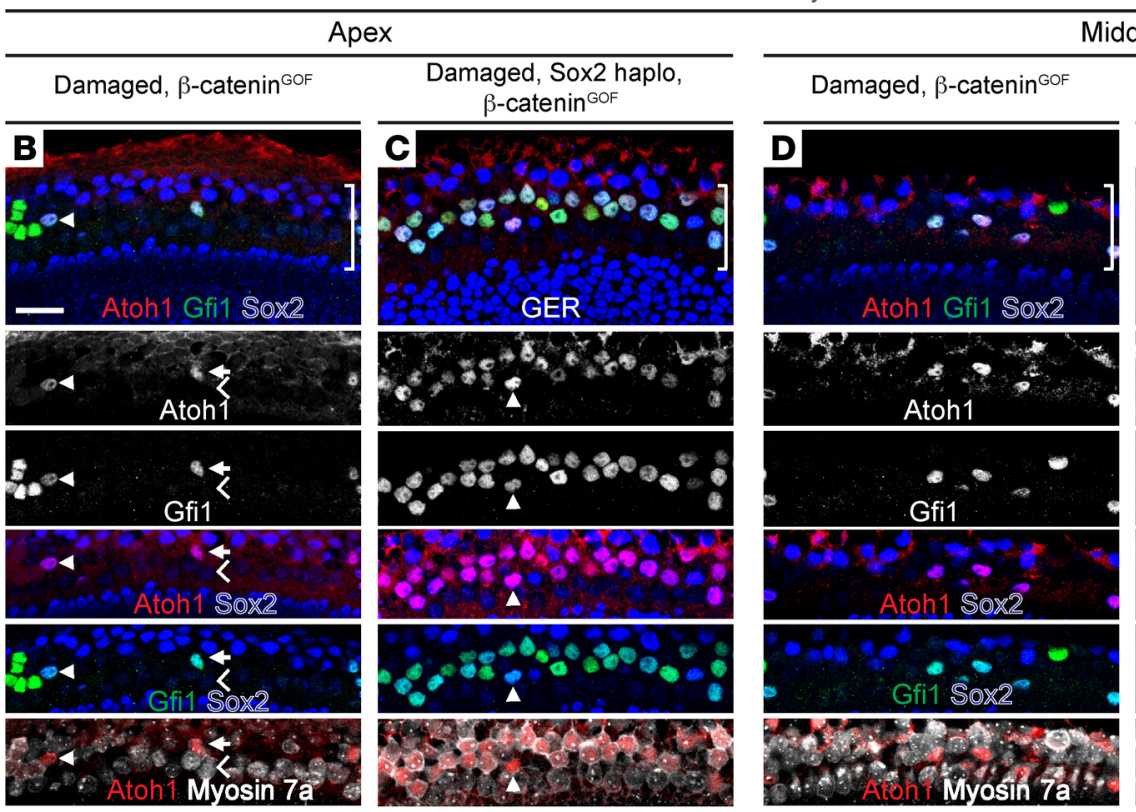

H
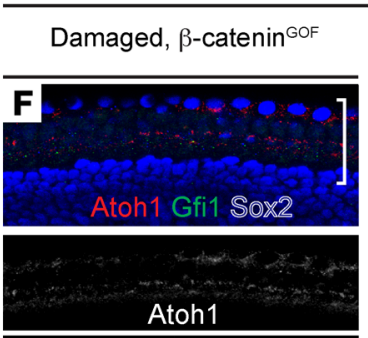

Gfi1

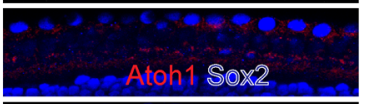

Gfi1 Sox?

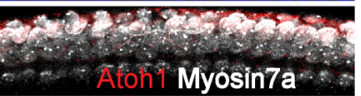

Base Damaged, Sox2 haplo, $\beta$-catenin ${ }^{\mathrm{GOF}}$

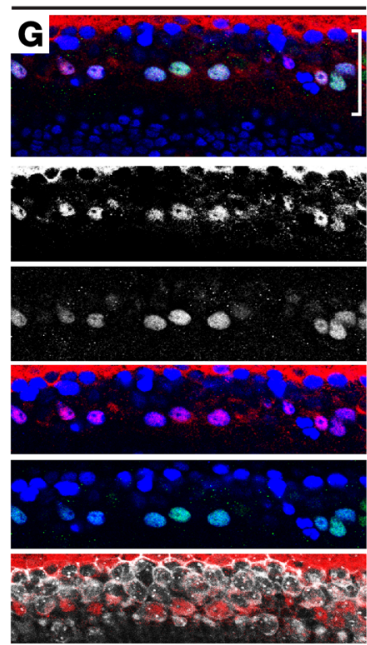

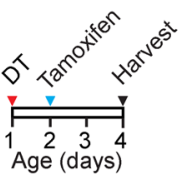


A

$\beta$-catenin ${ }^{\mathrm{GOF}}$

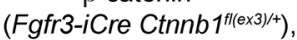
Sox2 haplo, $\beta$-catenin ${ }^{\mathrm{GOF}}$ (Sox $2^{\text {CreERT2/+ }}$ Ctnnb1 $1^{\text {H( }(\text { ex } 3 / /+}$ )

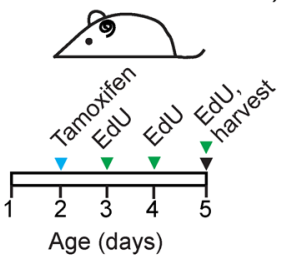

D

$\beta$-catenin ${ }^{\mathrm{GOF}}$

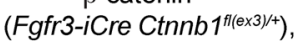
Conditional Sox2 haplo, $\beta$-catenin ${ }^{\mathrm{GOF}}$

(Fgfr3-iCre Sox $2^{f / /+}$ Ctnnb1 $1^{\text {fl(ex3)/+}}$ )

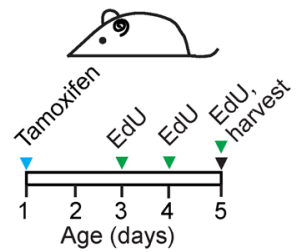

G

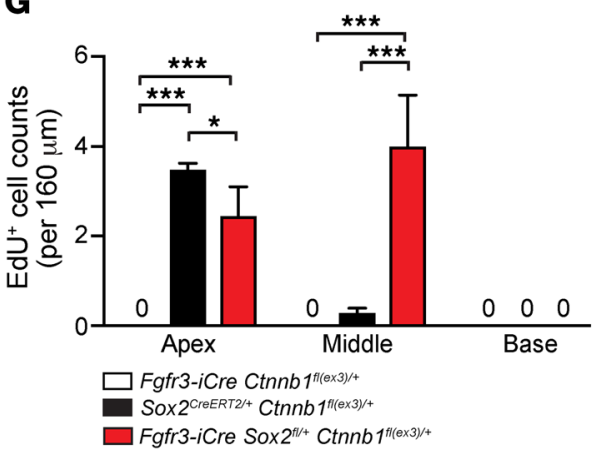

H

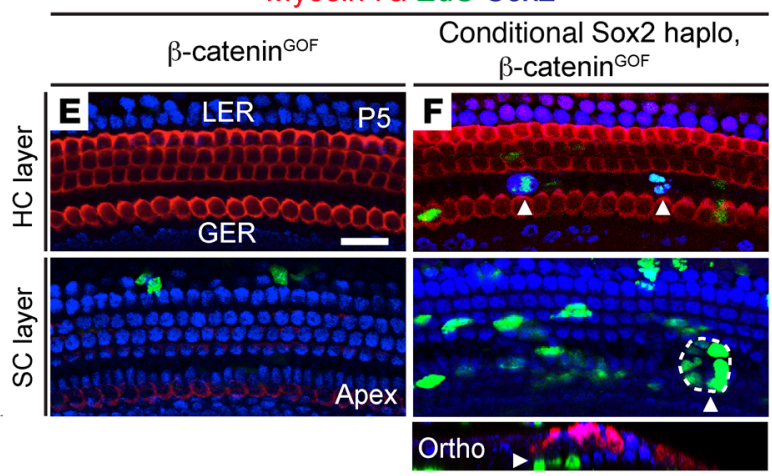

Myosin 7a EdU Sox2

$\beta$-catenin ${ }^{\mathrm{GOF}}$

(Fgfr3-iCre Ctnnb1 $1^{f((e \times 3) /+}$ ),

Sox2 haplo, $\beta$-catenin ${ }^{\mathrm{GOF}}$

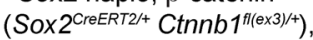

Conditional Sox2 haplo $\beta$-catenin ${ }^{\mathrm{OOF}}$

$\left(\right.$ Sox $2^{f / /+}$ Ctnnb $1^{1 /((2 \times 3) /++}$

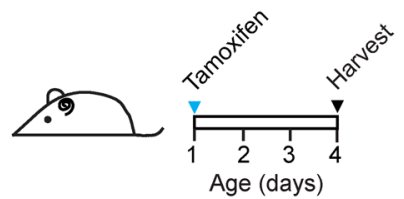

$\beta$-catenin ${ }^{\text {GOF }}$

Sox 2 haplo, $\beta$-catenin ${ }^{\text {GOF }}$ $\underset{\beta \text {-catenin }}{\text { Conditional Sox } 2 \text { haplo }}$
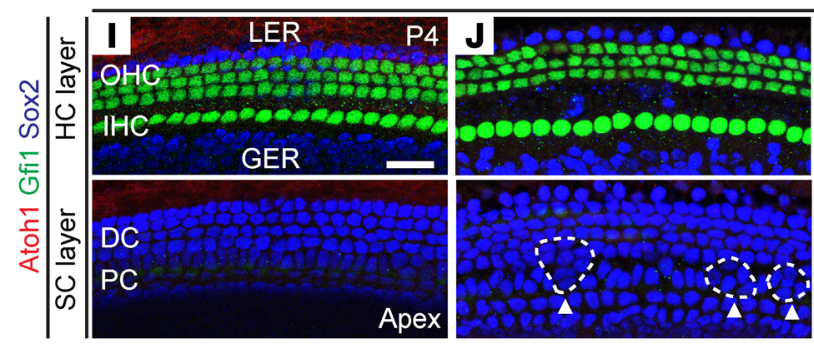

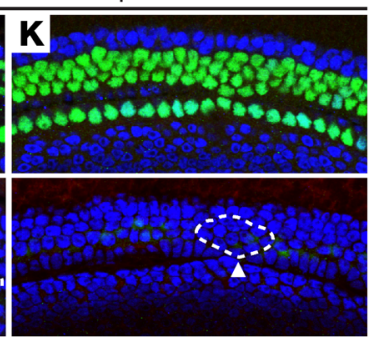

Figure 6. Sox2 haploinsufficiency acts as a permissive signal for $\beta$-catenin-induced proliferation in the undamaged neonatal cochlea. (A) Schematic of the experimental paradigm. Fgfr3-iCre $\left[\right.$ tnnb $7^{f(l e x 3) /+}$ and

Sox $2^{\text {[reERT2/++ }}$ tnnnb $^{\text {fl(ex3)/++ }}$ pups were given tamoxifen on $\mathrm{P} 2$, followed by daily administration of EdU (P3-P5), and cochleae were examined on P5. (B) No EdU ${ }^{+}$cells were detected in the organ of Corti in cochleae from Fgfr3-iCre Ctnnb ff(ex3)/+ $^{2}$ mice treated with tamoxifen on P2. (C) EdU+ supporting cells but not hair cells arranged as foci (dashed lines, arrowhead) were found in P5 Sox2 $2^{\text {CreERT2/+ }}$

Ctnnbifl(ex3)/+ cochleae. (D) Fgfr3-iCre Ctnnbiff(l(x)//+ and Fgfr3-iCre Sox $\mathrm{fl}^{\mathrm{fl}+} \mathrm{Ctn}$ $n b 7^{f(e x 3) /+}$ pups were given tamoxifen on P1, followed by EdU administration daily (P3-P5), and cochleae were examined on P5. (E) The apical turn of Fgfr3-iCre Ctnnb $7^{f(\text { (lex } x) /+}$ cochleae revealed no EdU+ hair cells or supporting cells. (F) Like Sox2 ${ }^{\text {CreERT2/+ }}$ Ctnnb $7^{\mathrm{fl}(\mathrm{ex}) /+}$ cochlea, but in contrast to Fgfr3-iCre Ctnnb $7^{f(\text { (ex3)/+ }}$ cochlea, $\mathrm{EdU}^{+}$cells arranged as clusters (dashed lines, arrowheads) were found in Fgfr3-iCre Ctnnb $7^{\mathrm{fl}(\mathrm{ex} 3 \mathrm{3}) /+}$ Sox $2^{\mathrm{fl} /+}$ cochlea (tamoxifen was given on P1). (G) Quantification of EdU ${ }^{+}$ cells. The differences in EdU+ cells between Sox $2^{\text {haplo }}$ and conditional Sox $2^{\text {haplo }}$ models can be attributed to the timing or degrees of Sox2 partial deletion. (H) Fgfr3-iCre Ctnnb ff(lex3)/+ Sox2 $2^{\text {(reERT2/+ }}$ Ctnnb $1^{f((-\times 3) /+}$, and Fgfr3-iCre Sox $2^{f / /+}$ Ctnnb $f^{f(1 /(x) /)^{+}}$pups were injected with tamoxifen on P1, and cochleae were harvested on P4. (I) Gfi1-labeled hair cells were present, but no Atoh1- or Gfi1-labeled Sox2 ${ }^{+}$supporting cells were detected in Fgfr3-iCre Ctnnb ff(ex3)/ cochlea. (J) No Atoh1- or Gfi1-labeled Sox2 ${ }^{+}$ supporting cells were detected in Sox $2^{\text {CreeRT2/+ }}$ Ctnnb $7^{f(\text { (ex3)/+ }}$ cochlea, although foci-like clusters were still noted in the pillar cell region (dashed lines, arrowheads). (K) Neither Atoh1- nor Gfi1-labeled transitional cells were detected in Fgfr3-iCre Ctnnbif(lex3)/+ Sox $2^{\mathrm{fl}++}$ cochlea (tamoxifen was administered on P1), although foci-like clusters could still be observed (dashed line, arrowhead). Data represent the mean \pm SD. ${ }^{*} P<0.05$ and ${ }^{* *} P<0.001$, by 1 -way ANOVA with Holm-Sidak multiple comparisons test. $n=3$. Scale bars: $20 \mu \mathrm{m}$.
(AG) (sisomicin with furosemide) (58) and DT (44) treatment (Figure 7A and Supplemental Figure 7A). After drug administration on P21, both damage paradigms led to significant hair cell loss by P28 (Figure 7, B-E). AGs caused primarily outer hair cell loss, and DT (Pou $43^{\text {DTR/+}}$ ) caused mainly inner hair cell loss. Both damage models showed significantly elevated ABR thresholds, with the AG model also showing higher DPOAE thresholds (Figure 7, F and G).

To test the effects of Sox $2^{\text {haplo }}$ and $\beta$-catenin ${ }^{\mathrm{GOF}}$ on damaged, mature cochlea, we examined the Fgfr3-iCre Ctnnb1 $1^{f(\text { (ex3)/+ }}$ and Sox $2^{\text {CreERT2/+ }}$ Ctnnb1 $^{f(\text { ex } 3) /+}$ mice $\left(\beta\right.$-catenin ${ }^{\mathrm{GOF}}$ alone and Sox $2^{\text {haplo }}$ and $\beta$-catenin ${ }^{\mathrm{GOF}}$, respectively) (Figure $7 \mathrm{H}$ ). Cochleae from

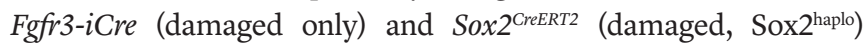
mice served as controls. Tamoxifen administration on P22 led to approximately $70.8 \% \pm 14.9 \% \mathrm{Cre}^{+}$supporting cells in Fgfr3-iCre Rosa $26^{\text {tdTomato/+}}$ cochleae (data not shown) and a decrease of $30.0 \% \pm$ 18.1\% in Ctnnb1 exon 3 mRNA levels in whole Fgfr3-iCre Ctnnb1 ${ }^{f(e x 3) /+}$ cochleae (Figure 7I), suggesting that activated Cre recombinase and $\beta$-catenin were effectively modified for stabilization.

In the AG- and DT-damaged cochleae, we detected no EdUlabeled cells within the organ of Corti in the $\beta$-catenin ${ }^{\mathrm{GOF}}$ or Sox $2^{\text {haplo, }}$, 
A

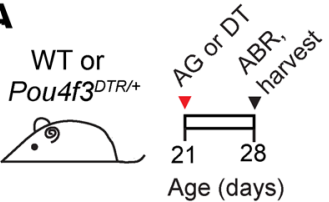

B Saline

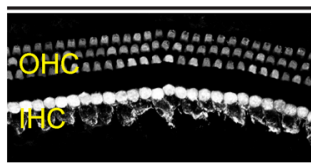

c

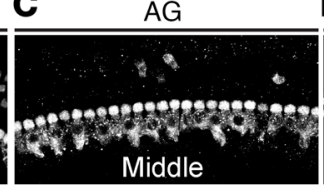

D

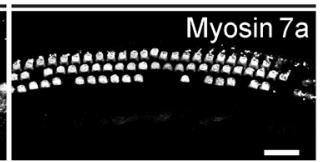

$\mathbf{E}$

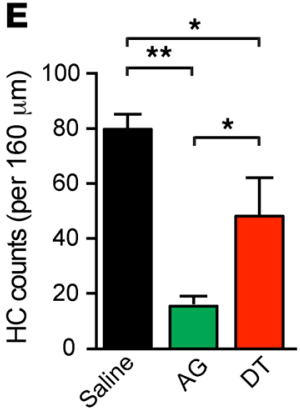

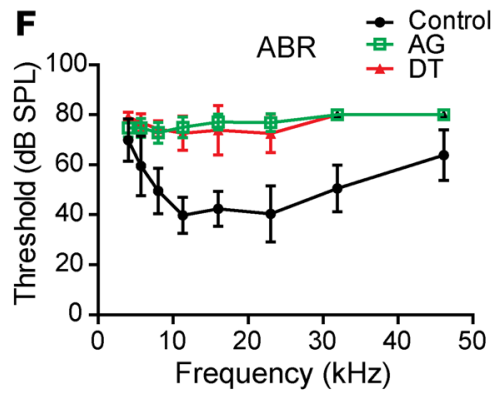

H Damaged, $\beta$-catenin ${ }^{\text {GOF }}$ (Pou4f3 ${ }^{\text {DTR/ }}$ Fgfr3-iCre Ctnnb1 $1^{\text {fl( }(\text { ex3)/+ }}$ ) Damaged, Sox2 haplo (Pou4f3 ${ }^{\text {DTR/+ }}$ Sox $2^{\text {CreERT2/+ }}$ Damaged, Sox 2 haplo, $\beta$-catenin ${ }^{\mathrm{GOF}}$ (Pou4f3 ${ }^{\text {DTR/+ }}$ Sox $2^{\text {CreERT2/+ }}$ Ctnnb1 $1^{f((\times 3) /+}$ )

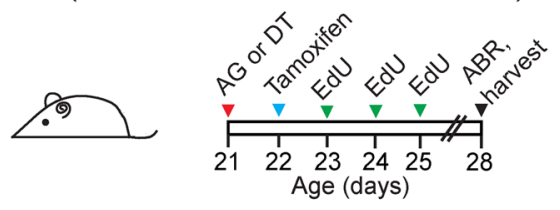

Myosin 7a EdU Sox2

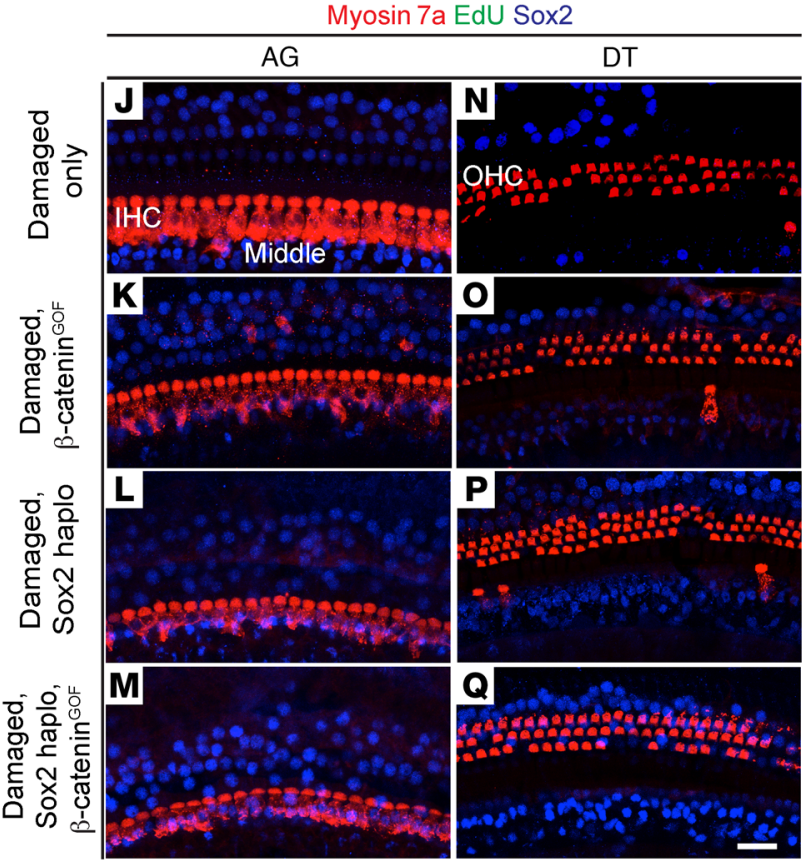

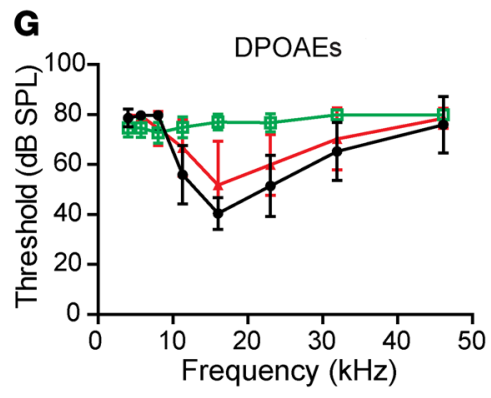

I
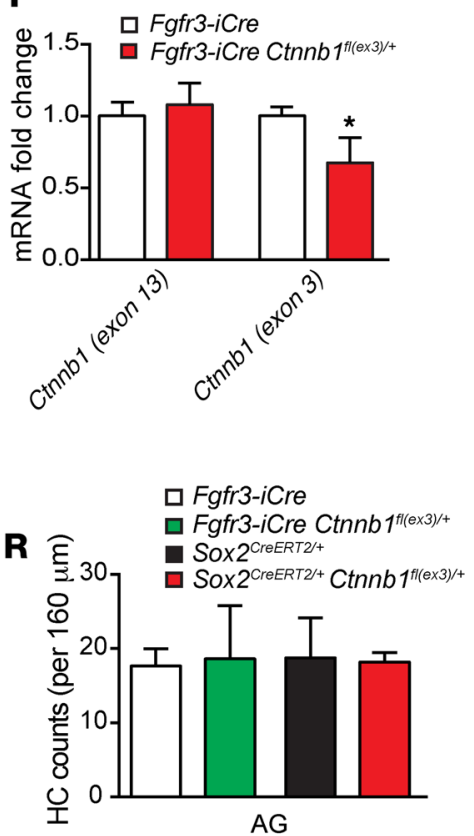

$\mathbf{S}$

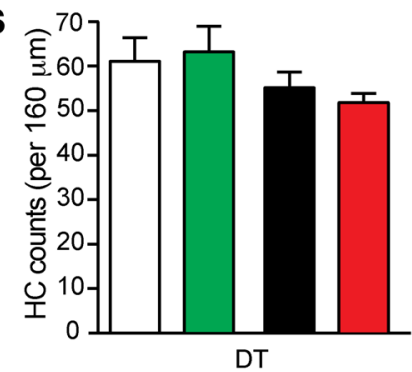

Figure 7. Sox2 haploinsufficiency and $\beta$-catenin stabilization do not induce mitotic hair cell regeneration in the damaged adult cochlea. (A) Schematic showing the use of AC (sisomicin combined with furosemide) or DT to damage the mature cochlea in WT and Pou $4 \mathrm{f3}^{\mathrm{DTR} /+}$ mice. (B-D) Saline-treated mice containing a full complement of myosin $7 a^{+}$ cochlear inner hair cells and outer hair cells. A loss of outer hair cells after AC treatment and of inner hair cells after DT treatment was observed. Some outer hair cell loss after DT treatment was observed.

(E) Quantification revealed a significant decrease in hair cell numbers in both damage paradigms. ( $\mathbf{F}$ and $\mathbf{G}$ ) ABR thresholds were significantly higher in both the AG- and DT-treated animals as compared with controls. A significant threshold shift was also observed in the DPOAEs in AG-treated animals, but not in the animals treated with DT. (H) Schematic of transgenic mouse models and experimental timeline. Cochleae were damaged on P21, tamoxifen was given on $\mathrm{P} 22$, followed by EdU administration from $\mathrm{P} 23$ to $\mathrm{P} 25$, and the animals were sacrificed after ABR on P28. (I) QPCR showing a significant reduction of Ctnnb1 (exon3) mRNA expression but not of Ctnnb1 (exon 13) mRNA expression in cochleae from Fgfr3-iCre Ctnnb ffl(lex3)/+ $^{2}$ mice. (J-M) No EdU + Sox2 $2^{+}$supporting cells or myosin $7 a^{+}$hair cells were detected after AC treatment in any of the genotypes examined. A persistent loss of outer hair cells was seen in these cochleae, without any new Sox $2^{+}$myosin $7 a^{+}$hair cells. (N-Q) There were no EdU ${ }^{+}$ hair cells or supporting cells after DT treatment or formation of new Sox $2^{+}$myosin $7 a^{+}$hair cells in any of the mouse cohorts. (R and $\mathbf{S}$ ) Quantification of hair cells in AGand DT-treated cochleae showing no change in hair cell numbers among genotypes. Data represent the mean \pm SD. ${ }^{*} P<0.05$ and ${ }^{* *} P$ $<0.01$, by 2 -tailed Student's $t$ test or 1-way ANOVA with Holm-Sidak multiple comparisons test. $n=$ 3-11. Scale bars: $20 \mu \mathrm{m}$. 
$\beta$-catenin ${ }^{\mathrm{GOF}}$ cochleae 1 week after damage (Figure 7, J-Q, and Supplemental Figure 7, B-Q), nor did we detect any EdU-labeled cells within the organ of Corti 3 weeks after damage (data not shown). Moreover, BrdU administered via the drinking water for 2 weeks (P23-P37) to AG-damaged Fgfr3-iCre Ctnnb1 ${ }^{f(l(x) 3) /+}$ mice also failed to label cells in the organ of Corti (Supplemental Figure 7, V-X). To assess for transitional cell formation, we immunostained cochleae from AG-treated, Sox $2^{\text {haplo }}, \beta$-catenin ${ }^{\mathrm{GOF}}\left(\right.$ Sox $2^{\text {CreERT2/+ }}$ Ctnnb $^{\text {flex3)/++}}$ ) mice and found no Atoh $1^{+}$supporting cells 4 days after damage (not shown). In addition, myosin $7 \mathrm{a}^{+}$hair cell counts did not differ among the 4 groups: (a) damaged-only control cochleae, (b) damaged and Sox 2 haplo cochleae, (c) damaged and $\beta$-catenin ${ }^{\mathrm{GOF}}$ cochleae, and (d) damaged, Sox $2^{\text {haplo }}$, $\beta$-catenin ${ }^{\mathrm{GOF}}$ cochleae) 1 week (Figure $7, \mathrm{R}$ and S) or 3 weeks after damage (data not shown). Lastly, ABR and DPOAE measurements revealed no differences in thresholds among damaged-only, damaged and $\beta$-catenin ${ }^{\mathrm{GOF}}$, and damaged, Sox $2^{\text {haplo }}, \beta$-catenin ${ }^{\mathrm{GOF}}$ animals 3 weeks after damage (Supplemental Figure 7, R-U). Thus, our data indicate that supporting cells in the damaged, mature cochlea are not competent to divide or form transitional cells in response to Sox $2^{\text {haplo }}$ and/or $\beta$-catenin ${ }^{\mathrm{GOF}}$.

Downstream targets of Sox2 haploinsufficiency. Our results so far suggest that Sox $2^{\text {haplo }}$ acts as a permissive signal for supporting cells to proliferate, form transitional cells, and be responsive to $\beta$-catenin in the damaged, neonatal cochlea but not in the damaged, mature cochlea. To gain insights into possible mechanisms governing this phenomenon, we measured mRNA expression of Notch (Hes5, Hes1, and Hey1) and Wnt (Axin2, Lef1, and Lgr5) target genes in 4 groups of cochleae from P5 and P25 mice: (a) undamaged WT; (b) undamaged, Sox $2^{\text {haplo }}$ (Sox $2^{\text {CreERT2/+ }}$ ); (c) damaged only (Pou $\left.4 f 3^{\text {DTR/+}}\right)$; and (d) damaged, Sox $2^{\text {haplo }}$ (Pou $4 f 3^{\text {DTR/+ }}$ Sox $2^{\text {CreERT2/+ }}$ ) (Figure 8A and Supplemental Figure 8A).

Both the neonatal and mature Sox $2^{\text {haplo }}$ cochleae expressed significantly lower levels of Sox $2(23.1 \% \pm 11.1 \%$ and $42.7 \% \pm 29.2 \%$, respectively) when compared with levels in WT tissues (Figure 8, $\mathrm{B}$ and C). We also detected a significant reduction in Sox2 levels in the damaged-only (Pou $4 \mathrm{f}^{\mathrm{DTR} /+}, 29.1 \% \pm 16.7 \%$ ) cochleae and damaged, Sox $2^{\text {haplo }}$ (Pou $43^{\text {DTR/+ }}$ Sox $2^{\text {CreERT2/+ }}, 21.4 \% \pm 6.1 \%$ ) cochleae compared with levels in WT control cochleae (Figure 8B). By contrast, Sox2 levels did not significantly change after damage in the mature cochlea (Figure $8 \mathrm{C}$ ).

Neonatal cochleae from Sox $2^{\text {CreERT2/+ }}$ mice had a significant reduction in levels of the Notch target gene Hes5 $(47.8 \% \pm 29.9 \%)$ relative to levels in WT control cochleae (Figure 8D). This decrease was also detected using semiquantitative in situ experiments on sections from Sox $2^{\mathrm{CrERT2} /{ }^{+}}$and WT cochleae (Supplemental Figure 9, A-F). After DT damage alone (Pou $\left.4 \mathrm{f}^{\mathrm{DTR} /+}\right)$ or when combined with Sox $2^{\text {haplo }}$ (Pou $43^{\text {DTR/+ }}$ Sox $2^{\text {CreERT2/+}}$ ), we also observed a significant decrease in Hes5 expression $(25.4 \% \pm 10.3 \%$ and $31.3 \%$ $\pm 10.6 \%$, respectively) (Figure 8D). However, no change in Hes5 expression was detected in the mature cochlea (Figure 8E). Moreover, we observed no changes in the levels of expression of other Notch target genes (Hes1 and Hey1) in the neonatal or mature cochleae (Supplemental Figure 8, B-E). We also found no significant changes in the expression of Wnt target genes (Axin2, Lgr5, and Lef1) in the 4 groups of neonatal and mature cochleae examined (Supplemental Figure 8, F-K). These results suggest that decreased expression in components of the Notch pathway may mediate the permissive signals conferred by Sox $2^{\text {haplo }}$ or damage to induce proliferation, the formation of transitional cells, and responsiveness to $\beta$-catenin ${ }^{\mathrm{GOF}}$.

\section{Discussion}

Sox 2 directs the cell cycle and cell fate during the development of diverse tissue types. Complete and partial Sox 2 deficiencies cause multiorgan defects including anophthalmia, epilepsy, and trachea-esophageal, cochlear, and genital anomalies in mice and humans (10, 11, 17-20). Moreover, some regenerating tissues are sensitive to Sox2 dosage and are dependent on Sox2 activation $(10,17,27)$. Our study shows that Sox2 haploinsufficiency allows essentially normal development of the cochlea. Furthermore, in contrast to the previous notion that Sox2 upregulation promotes tissue regeneration, Sox 2 haploinsufficiency primes the cochlea to regenerate in vivo.

Competence and domains of tissue regeneration. In the avian cochlea, 2 mechanisms mediate hair cell regeneration in distinct regions of the organ: nonmitotic, direct transdifferentiation in the abneural region and mitotic regeneration in the neural domain (59). While the mature mammalian cochlea does not regenerate, fate-mapping studies demonstrate that supporting cells in the neonatal cochlea spontaneously proliferate and form new hair cells after damage $(43,45)$. Cox and colleagues showed that proliferation primarily occurred in the apical region, while the fatemapped, immature hair cells were present in all 3 turns of the neonatal cochlea. Given the results of these studies, we hypothesized that proliferation competence and phenotypic conversion (which we characterize as transitional cell formation) are distinct and differentially regulated.

Several findings in our study support the notion that cell proliferation and transitional cell formation are differentially governed. First, while mitotic cells in the Sox $2^{\text {haplo }}$ cochlea mainly resided in the apical turn, we found ectopic hair cells in all 3 cochlear turns. After damage, mitotic regeneration occurred primarily in the apical turn, with transitional (Atoh1 ${ }^{+}$) cell formation observed more broadly in the apical and middle turns (Figure 8F). Thus, as with Sox $2^{\text {haplo }}$, damage confers competence in supporting cells to both proliferate and form transitional cells. Moreover, the effects of Sox $2^{\text {haplo }}$ and damage appear additive in conferring to supporting cells the competence to mitotically regenerate and form transitional cells. Building on previous studies showing that Sox2 antagonizes hair cell specification in the embryonic cochlea (11) and inhibits proliferation (60), the current results unveil roles for Sox $2^{\text {haplo }}$ as a positive modulator of mitotic hair cell regeneration and transition cell formation in the damaged postnatal cochlea. However, Sox 2 is required during hair cell regeneration in the zebrafish inner ear (61), and whether Sox2 is similarly indispensable during mammalian hair cell regeneration remains to be determined.

Competence to respond to Wnt activation. In contrast to previous studies $(54,55)$, our results indicate that postnatal cochlear supporting cells in a resting, undamaged state are not competent to proliferate or form ectopic hair/transitional cells upon Wnt activation. Rather, damage or Sox $2^{\text {haplo }}$ confers to supporting cells the competence to proliferate in response to Wnt activation. Further, the competence to form additional transitional cells in response to Wnt activation requires the presence of both 
A

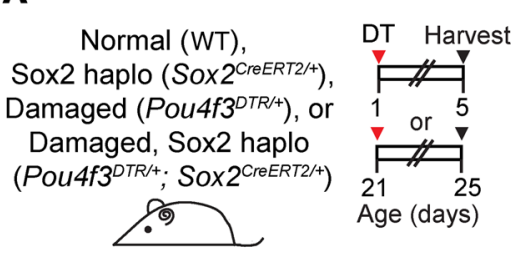

B

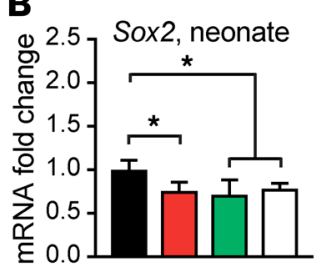

C

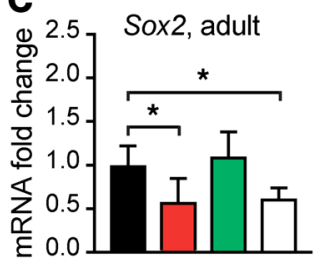

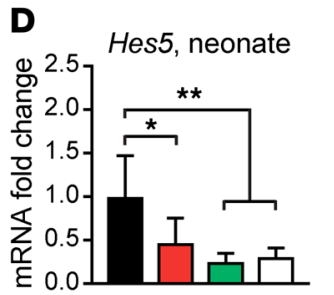

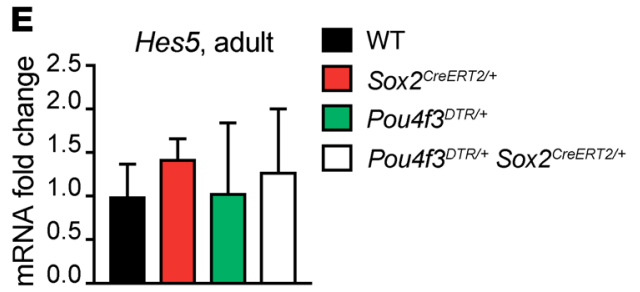

$\mathbf{F}$

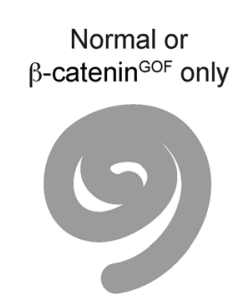

EdU None

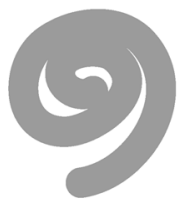

Atoh1
No damage Sox2 haplo $\beta$-catenin ${ }^{\text {GOF }}$

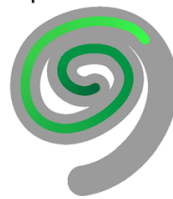

Moderate

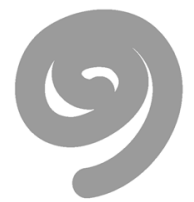

None
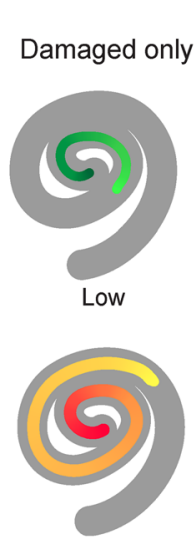

Low

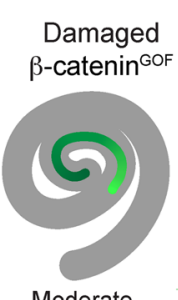

Moderate

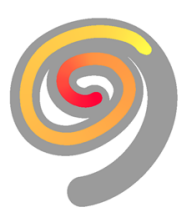

Low

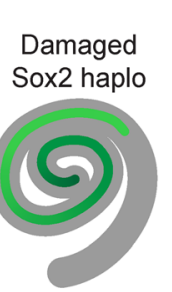

Moderate

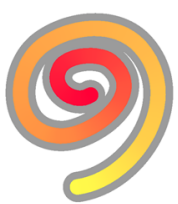

Moderate
Damaged

Sox2 haplo $\beta$-catenin ${ }^{\mathrm{GOF}}$

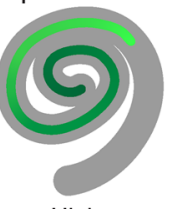

High

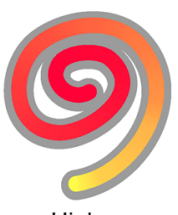

High
Figure 8. qPCR of Notch and Wnt target genes in damaged and Sox2-haploinsufficient cochleae. (A) Neonatal (P1) and mature (P21) WT, Sox $2^{\text {CreERT2/+ }}$, Pou $4 \mathrm{f}^{\mathrm{DTR} /+}$, and Pou $4 f 3^{\text {DTR/+ }}$ Sox2 $2^{\text {CreERT2/+ }}$ mice were treated with $\mathrm{DT}$, and cochleae were collected 4 days later. (B) A significant decrease was detected in Sox2 expression levels in the neonatal Sox2 $2^{\text {CreERT2/+ }}$, Pou $4 f 3^{\text {DTR/+ }}$, and Pou $4 f 3^{\text {DTR/+ }}$ Sox $2^{\text {CreERT2/+ }}$ cochleae relative to levels in WT controls. (C) Only the mature Sox $2^{\text {CreERT2/+ }}$ and Pou $43^{\text {DTR/+ Sox }} 2^{\text {CreERT2/+ }}$ cochleae had lower Sox2 levels than those detected in control cochleae. (D) A significant decrease in the levels of the Notch target gene Hes 5 was detected in Sox2 $2^{\text {CreERT2/+ }}$, Pou $4 f 3^{\text {DTR/+ }}$, and Pou $4 f 3^{\text {DTR/+ }}$ Sox $2^{\text {CreERT2/+ }}$ cochleae relative to levels in WT cochleae on P5. (E) When measured on $\mathrm{P} 25$, no significant changes in the expression levels of Hes5 were seen relative to WT cochleae. Expression levels of Sox2 and Hes5 in the mature WT cochleae were lower than levels in P5 WT cochleae. (F) Schematics depicting the extent and patterns of proliferation and Atoh1 ${ }^{+}$transitional cells under various defined conditions. Darker colors represent more robust proliferation or the formation of transitional cells. (G) Proposed model of Sox2 and damage coordination in regulating mitotic regeneration, transitional cell formation, and Wnt responsiveness. ${ }^{*} P<0.05$ and ${ }^{*} P$ $<0.01$, by 1 -way ANOVA with HolmSidak multiple comparisons test. $n=4$.

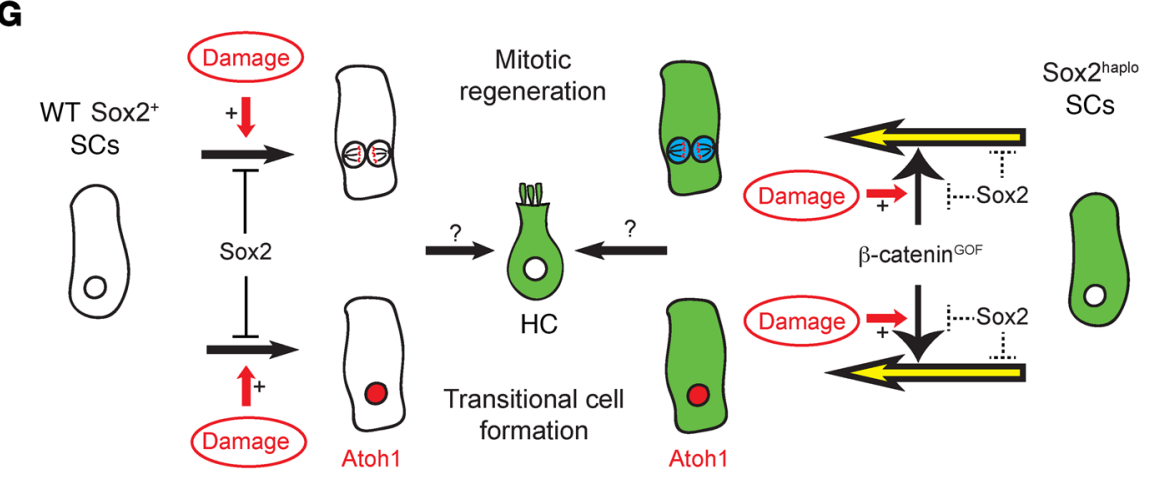

damage and Sox $2^{\text {haplo }}$. These findings lend additional evidence for the differential regulation of mitotic regeneration and transitional cell formation.

While the context-dependent effects of Wnt activation are known (62), the mechanisms dictating the permissiveness to respond to $\beta$-catenin activation remain poorly understood. A potential mechanism is the differential inhibition of Wnt signaling, a finding noted in intestinal polyposis and hepatic carcinogenesis (63). Several Sox family transcription factors directly interact with $\beta$-catenin to repress Wnt transcriptional responses, while others can enhance Wnt target gene expression (64). Sox2 represses proliferation in Wnt-driven gastric adenoma formation (65) and cancer (66), but in other contexts such as breast cancer, it stimulates $\beta$-cat- enin-induced proliferation (67). This study posits a model in which Sox 2 represses the mitogenic and prosensory effects of $\beta$-catenin activation, possibly via activation of the Notch signaling effector Hes5. Although we did not detect a significant change in defined Wnt target genes in Sox 2 haplo or damaged cochleae, it is also possible that decreased Sox 2 expression causes a small degree of disinhibition of Wnt signaling and directly facilitates the effects induced by $\beta$-catenin stabilization. In support of our finding of Notch signaling involvement, Sox2 regulates retinal progenitor cell proliferation/ differentiation in a dose-dependent manner via Notch signaling (10). Moreover, Notch1 deletion enhances $\beta$-catenin-induced mitotic generation of hair cells in the neonatal cochlea (68). Like Sox2, Hes 5 suppresses Atoh1 during cochlear development $(69,70)$, 
and Notch inhibition increases Atoh1 expression and hair cell formation in undamaged (71-73) and damaged neonatal cochleae (74).

In the postnatal cochlea, Hes 5 is highly expressed in Sox $2^{+}$supporting cells and becomes downregulated in the mature cochlea (75-77). The most notable difference between the neonatal and mature cochleae is that the former had lower expression of Hes 5 as a result of Sox $2^{\text {haplo }}$ or damage. By contrast, Hes 5 and other Notch target genes were expressed at low levels and were not altered as a result of Sox 2 haplo or damage in the mature cochlea, suggesting that they play a significant role in restricting hair cell regeneration in the neonatal, but not the mature cochlea. Thus, Sox $2^{\text {haplo }}$ and damage may coordinate to dictate mitotic regeneration, transitional cell formation, and Wnt responsiveness via Notch signaling in the neonatal cochlea, but not in the mature cochlea (Figure 8G) because of age-related changes of these factors. Our results are in agreement with previous reports noting a decline of Hes 5 expression in the maturing cochlea $(72,75)$. Furthermore, Hes 5 and Atoh 1 expression remained low in the noise-damaged mature cochlea (77). Another important consideration is the largely unknown network of transcription factors that act in concert with Sox2 and Hes5 in the neonatal and mature cochlea. Moreover, gene silencing by epigenetic factors occurs as the cochlea develops and matures (78), thus a more comprehensive investigation in these areas, including further study of the roles of Hes5, should be of interest and may help guide future therapy using Sox 2 and Wnt signaling to regenerate the mature cochlea.

Mouse models to study regeneration. The results of this study underscore the potential confounding effects from haploinsufficiency when using knockin mouse models (79). For example, the Foxg1-Cre mouse line can have brain deficits that probably stem from alterations in proliferation, possibly as a result of haploinsufficiency (80-82). In the same vein, the differences between our results and those of previous studies, in which $\beta$-catenin stabilization induced proliferation in the nondamaged neonatal cochlea, can be attributed to the knockin mouse lines used (Sox $2^{\text {CreERT2/+ }}$ and $\left.\operatorname{Lgr}^{\text {CreERT2/+}}\right)(54,55)$. Moreover, the proliferative cells observed after $\beta$-catenin stabilization in Lgr5 ${ }^{+}$cells in the neonatal cochlea may have originated from inner phalangeal cells medial to the inner hair cells. While the robust proliferation observed in the neonatal Sox $2^{\text {CreERT2/+ }}$ cochlea is affected by Sox 2 haploinsufficiency $(55,68,83)$, one should also consider the differences in experimental paradigms used and the supporting cell subtypes examined.

In summary, our results introduce a model system for mammalian hair cell regeneration, in which Sox2 haploinsufficiency enhances regeneration, and reveal the cooperative roles between Sox2 haploinsufficiency and damage in governing mitosis, differentiation, and Wnt responsiveness of hair cell precursors in the neonatal cochlea, probably via components of Notch signaling. These findings illustrate the differential responsiveness of cochlear supporting cells as hair cell progenitors in resting and damaged states. Moreover, these findings demonstrate the possibility of priming supporting cells, without hindering their normal function, to enhance their regenerative ability in response to damage and/or adjuvant therapy such as Wnt activation. Thus, this study should provide insights that will help guide the future design of combinatorial approaches for stimulating mammalian cochlear regeneration to reverse hearing loss.

\section{Methods}

Mice. The following mouse strains were used: Sox $2^{f /+}$ (The Jackson Laboratory; stock 13093) (84); Fgfr3-iCre (gift of W. Richardson, Wolfson Institute for Biomedical Research, University College London, Lon-

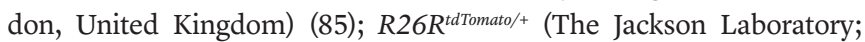
stock 7914) (86); Ctnnb1 ${ }^{f(e x 3) /+}$ (gift of M. Taketo, Kyoto University, Kyoto, Japan) (53); Sox $2^{\text {GFP/+ }}$ (The Jackson Laboratory; stock 17592) (21); Pou $43^{\text {DTR/+ }}$ (gift of E. Rubel, Department of Otolaryngology - Head and Neck Surgery, University of Washington, Seattle, Washington,

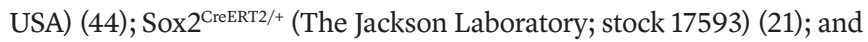
Atoh $1^{\text {GFP/+ }}$ (The Jackson Laboratory; stock 13593) (50). Mice of both sexes were used. To induce Cre recombinase activity, tamoxifen (dissolved in corn oil; MilliporeSigma) was injected i.p. into neonatal (0.075 $\mathrm{mg} / \mathrm{g})$ and adult $(0.225 \mathrm{mg} / \mathrm{g})$ mice. In neonatal mice, hair cell loss was induced on P1 by i.p. administration of DT ( $6.25 \mathrm{ng} / \mathrm{g}$; MilliporeSigma). In WT mice, damage was induced on P21 by a combined treatment with the AG sisomicin sulfate (i.p., $200 \mathrm{mg} / \mathrm{kg}$; OChem) and the loop diuretic furosemide (i.p., $300 \mathrm{mg} / \mathrm{kg}$; Novaplus) (58). P21 Pou $4 \mathrm{f}^{\mathrm{DTR} /+}$ mice were administered DT (i.p., $6.25 \mathrm{mg} / \mathrm{kg}$ ), EdU (i.p., $25 \mathrm{mg} / \mathrm{kg}$; Invitrogen, Thermo Fisher Scientific), or BrdU (1 mg/ml in the drinking water; Invitrogen, Thermo Fisher Scientific).

Genotyping and $q P C R$. A genomic DNA template was produced by adding $180 \mu \mathrm{l}$ of $50 \mathrm{mM} \mathrm{NaOH}$ to tissue biopsies and incubating at $98^{\circ} \mathrm{C}$ for 1 hour, at which point $20 \mu \mathrm{l}$ of $1 \mathrm{M}$ Tris- $\mathrm{HCl}$ was added. The primers used were as follows: Cre mutant, forward (5'-GCGGTCTGCCAGTAAAAACTATC-3'), Cre mutant, reverse (5'-GTGAAACAGCATTGCTGTCACTT-3'); Cre WT, forward (5'-CTAGGCCACAGAATTGAAAGATCT-3'), Cre WT, reverse (5'-GTAGGTGGAAATTCTAGCATCC-3'); Ctnnb $1^{f(e \times 3) /+}$, forward (5'-AAGGTAGAGTGATGAAAGTTG-3'), Ctnnb $1^{f(e x 3) /+}$, reverse (5'-CACCATGTCCTCTGTCTATTC-3'); Fgfr3-iCre, forward (5'-GAGGGACTACCTCCTGTACC-3') and Fgfr3-iCre, reverse (5'-TGCCCAGACTCATCCTTGGC-3'); R26R tdTomato/+ $^{\text {tTutant, for- }}$ ward (5'-CTGTTCCTGTACGGCATGG-3'), R26R tdTomato/+ $^{\text {tdont, }}$ reverse (5'-GGCATTAAAGCAGCGTATCC-3'); $R 26 R^{\text {tdTomato/+ }}$ WT, forward ( $5^{\prime}$-AAGGGAGCTGCAGTGGAGTA-3'), R26 ${ }^{\text {tdTomato/+ }}$ WT, reverse ( $5^{\prime}$-CCGAAAATCTGTGGGAAGTC- $\left.3^{\prime}\right)$; Pou $43^{\text {DTR/++}}$, forward (5'-GTCAAAAAATGTGCCTTAGAGCGG-3'), Pou $4 \mathrm{f}^{\text {DTR/+}}$, reverse (5'-CCGACGGCAGCAGCTTCATGGTC- $\left.3^{\prime}\right)$; Pou $43^{\text {DTR/+ }}$ WT, forward (5'-CACTTGGAGCGCGGAGAGCTAG-3'), Pou $43^{\text {DTR/+ }}$ reverse (5'-CCGACGGCAGCAGCTTCATGGTC-3'); So $2^{f /++}$, forward (5'-TGGAATCAGGCTGCCGAGAATCC-3'), So $2^{f l /+}$, reverse (5'-TCGTTCTGGCAACAAGTGCTAAAGC- $\left.3^{\prime}\right)$; So $\times 2^{\text {GFP/+ }}$ mutant, forward (5'-AAGTTCATCTGCACCACCG-3'), Sox $2^{\text {GFP/+ }}$ mutant, reverse (5'-TCCTTGAAGAAGATGGTGCG-3'); So $2^{\text {GFP/+ }}$ WT, forward (5'-CGTGATCTGCAACTCCAGTC- $\left.3^{\prime}\right)$, Sox $2^{\text {GFP/+ }}$ WT, reverse (5'-GGAGCGGGAgAAATGGATATG-3'); Atoh1 ${ }^{\text {GFP/+ }}$ WT, forward (5'-AGGGTCAGCTTGCCGTAGGT-3'); and Atoh $1^{\text {GFP/+ }}$ mutant, forward (5'-GCGATGATGGCACAGAAGG-3'), Atoh $1^{\text {GFP } /+}$ mutant, reverse (5'-GAAGGGCATTTGGTTGTCTCAG-3').

For qPCR, total RNA was isolated with an RNeasy Mini Extraction Kit (QIAGEN), and then cDNA was synthesized using SuperScript VILO cDNA Kit (Invitrogen, Thermo Fisher Scientific). TaqMan Fast Advanced Mix and Assays (Applied Biosystems) were used to perform qPCR reactions on a CFX Connect Real-Time System (Bio-Rad). All qPCR reactions were performed in triplicate, and the relative quantification of gene expression was analyzed using the $\Delta \Delta \mathrm{Ct}$ method (87). These 
results were normalized to an averaged value of 3 housekeeping genes (B2M, Hprt, and RplpO for Sox $2^{\text {CreERT2/+ }}$ or GAPDH, Hprt, and Snrpd3 for $C$ tnnb1 ${ }^{f(e x 3) /+}$ experiments) (31). The following probes were used: Axin2 (Mm00443610_m1); B2M (MM00437762_m1); Ctnnb1 (exon 3) (Mm00483029_g1); Ctnnb1 (exon 13) Mm00483039_m1; GAPDH (Mm9999999915_g1); Hes1 (Mm01342805_m1), Hes5 (Mm00439311 g1); Hey1 (mm00468865_m1); Hprt (Mm01545399_m1); Lef1 (Mm00550265_m1); Lgr5 (Mm00438905_m1); RplpO (Mm00725448_ s1); Snrpd3 (Mm01151268_m1); and Sox2 (Mm03053810_s1).

Auditory measurements. Auditory brainstem responses and DPOAEs were measured as previously described (58). Briefly, mice were anesthetized with i.p. injection of $100 \mathrm{mg} / \mathrm{kg}$ ketamine and 10 $\mathrm{mg} / \mathrm{kg}$ xylazine. Auditory responses were recorded from a needle electrode placed inferiorly to the tympanic bulla, referenced to an electrode on the vertex of the head, with a ground electrode placed into the hind limb. Tone pip stimuli were delivered in frequencies ranging from 4 to $46 \mathrm{kHZ}(4.0,5.7,8.0,11.3,16.0,23.0,31.9$, and $46.1 \mathrm{kHZ})$ up to a sound pressure level (SPL) of $80 \mathrm{~dB}$, in 10-dB steps. At each frequency and intensity, 260 trials were conducted and averaged. DPOAEs were measured by a probe-tip microphone placed into the auditory canal. The sound stimuli used to elicit the DPOAE were two 1-second sine wave tones of differing frequencies $(\mathrm{F} 2=1.22 \times \mathrm{F} 2)$. F2 ranged from 4 to $46 \mathrm{kHz}$, and the 2 tones were stepped up from 20 to $80 \mathrm{~dB}$ SPL in $10-\mathrm{dB}$ increments. The amplitude of the cubic distortion product was $2 \times$ F1-F2. The threshold was calculated as a DPOAE of greater than 5 $\mathrm{dB}$ SPL and 2 SDs above the noise floor for each frequency. For analysis of $\mathrm{ABR}$ and DPOAE responses, a lack of a response was designated at the highest sound level, $80 \mathrm{~dB}$ SPL.

IHC. Neonatal and adult cochleae were isolated and fix in $4 \%$ paraformaldehyde in PBS for 30 to 40 minutes (in PBS, pH7.4; Electron Microscopy Services) for further processing. Adult tissues were decalcified in $120 \mathrm{mM}$ EDTA for 24 to 36 hours at $4^{\circ} \mathrm{C}$. Tissues from mice of both ages were dissected into 3 turns, with the Reissner's and tectorial membranes and stria vascularis removed. Tissues were then washed with $0.1 \%$ Triton X-100 (in PBS) 3 times for 5 to 10 minutes each and blocked with $5 \%$ donkey serum, $0.1 \%$ Triton X-100, 1\% BSA, and $0.02 \%$ sodium azide $\left(\mathrm{NaN}_{3}\right)$ in $\mathrm{PBS}$ at $\mathrm{pH} 7.4$ for 1 hour at room temperature. Next tissues were incubated with primary antibodies in the same blocking solution overnight at $4^{\circ} \mathrm{C}$. The following day, tissues were washed with $0.1 \%$ Triton X-100 in PBS and incubated with secondary antibodies diluted in PBS containing 0.1\% Triton X-100, 1\% BSA, and $0.02 \% \mathrm{NaN}_{3}$ for 2 hours at room temperature. After washing with PBS 3 times for 10 minutes, tissues were mounted in Antifade Fluorescence Mounting Medium (Dako) and coverslipped.

The following antibodies were used: rabbit anti-myosin 7a (1:500 to 1:1,000; Proteus Bioscience; catalog 25-6790); mouse anti-myosin 7a (1:500; Developmental Studies Hybridoma Bank, University of Iowa; catalog 138-1-c); goat anti-Sox2 (1:200 to 1:400; Santa Cruz Biotechnology; catalog SC17320); rabbit anti-Atoh1 (1:1,000; Proteintech; catalog 21215-1AP); guinea pig anti-Gfi1 (1:1,000; gift of H. Bellen, Department of Molecular and Human Genetics, Baylor College of Medicine, Houston, Texas, USA) (32); rabbit anti-Ki67 (1:500; Abcam; catalog ab16667); mouse anti-BrdU (1:500; Thermo Fisher Scientific; catalog B35128); rabbit anti-Lef1 (1:400; Cell Signaling Technology; catalog 2230s); and Alexa Fluor donkey anti-goat 488 (A11055), Alexa Fluor donkey anti-goat 546 (A11056), Alexa Fluor donkey anti-goat 647 (A21447), Alexa Fluor donkey anti-mouse 488 (A21202), Alexa
Fluor donkey anti-mouse 546 (A10036), Alexa Fluor donkey antimouse 647 (A31571), Alexa Fluor donkey anti-rabbit 488 (A21206, Alexa Fluor donkey anti-rabbit 546 (A10040), Alexa Fluor donkey anti-rabbit 647 (A31573) secondary antibodies (1:250 to 1:500; Life Technologies, Thermo Fisher Scientific). Fluoresecence-conjugated phalloidin (1:1,000; Invitrogen, Thermo Fisher Scientific; catalog A22283); DAPI (1:10,000; Invitrogen, Thermo Fisher Scientific; catalogD1306); and an Alexa Fluor 555 or 647 EdU Detection Kit (Invitrogen, Thermo Fisher Scientific; catalog C10338/C10340). To assess proliferation in cochleae from mice treated with BrdU following secondary antibody incubation, tissues were washed with PBS and repermeabilized, after which tissues were incubated with $2 \mathrm{~N} \mathrm{HCl}$ in $0.05 \%$ Triton X-100 for 1 hour at room temperature. The tissues were then washed with PBS and blocked and immunostained with anti-BrdU as outlined above.

Whole mounts were imaged as Z-stacks on a Zeiss LSM700 confocal microscope, and images were captured using Zen Software (Carl Zeiss) and analyzed with Image (NIH) and Photoshop CS6 (Adobe Systems).

ISH. Tissues were harvested on ice, fixed in 4\% PFA overnight, and then embedded for cryosectioning as described previously (57). Briefly, tissues were sequentially submerged in $10 \%, 20 \%$, and $30 \%$ sucrose prior to tissue embedment and freezing in 100\% OCT (Sakura). Tissue sections were hybridized with commercial probes from Advanced Cell Diagnostics according to the manufacturer's instructions for fixed, frozen sections with colorimetric detection (88). Briefly, sections were washed in $1 \times$ PBS for 5 minutes and then treated with $\mathrm{H}_{2} \mathrm{O}_{2}$ for 10 minutes. Next, sections were permeabilized using target retrieval reagent and proteinase before hybridization. The following Advanced Cell Diagnostics probes were used: Hes5 (catalog 400991), DapB (catalog 310043), Polr2a (catalog 310451), and Sox2 (catalog 401041). WT and Sox $2^{\text {CreERT2/+ }}$ cochleae were processed in parallel, with sections collected on the same slide and subjected to mRNA detection under identical conditions.

Cell quantification. For cell counting, confocal images were analyzed using ImageJ. To quantify EdU-labeled cells and ectopic hair cells in neonatal cochleae, $Z$-stack images were taken of the whole cochleae, which were divided into apical, middle, and basal turns of equal length, and cells were counted from stacks using ImageJ. Other cell counts in the neonatal and the adult cochleae were performed on representative $Z$-stack images of individual turns.

Statistics. Data were analyzed using Microsoft Excel and GraphPad Prism Version 7 (GraphPad Software). A 2-tailed Student's $t$ test or ANOVA with post hoc testing was used to calculate statistical significance. A $P$ value of less than 0.05 was considered statistically significant. Data shown in the figures represent the mean \pm SD. For all experiments, $n$ values represent the number of animals examined.

Study approval. All experimental protocols were approved by the IACUC of Stanford University.

\section{Author contributions}

PJA, YD, and AGC designed the studies. PJA, YD, SG, WL, EHN, and TU conducted the experiments. PJA, YD, and AGC analyzed the data and wrote the manuscript.

\section{Acknowledgments}

We are deeply grateful to the members of our laboratory; R. Nusse, S. Heller, and B. Hartman (all from Stanford Univer- 
sity, Stanford, CA, USA) for insightful comments and fruitful discussions of the manuscript; J. Eide, K. Han, Q. Wang, T. Jan, and W. Dong (all from Stanford University, Stanford, CA, USA) for excellent technical support; E. Rubel (University of Washington, Seattle, WA, USA) for sharing Pou4f3-DTR mice; W. Richardson (University College London, London, United Kingdom) for sharing Fgfr3-iCre mice; M. Taketo (Kyoto University, Kyoto, Japan) for sharing Ctnnb1-flox(ex3) mice; and H. Bellen (Baylor College of Medicine, Houston, TX, USA) for the providing Gfi1 antibodies. This work was supported by the Lucile Packard Foundation for Children's Health, Stanford; the NIH National Center for Advancing Translational Sciences - Clinical and Translational Science Awards (NCATS-CTSA) program (UL1TR001085); the Child Health Research Insti- tute of Stanford University; a Garnett Passe and Rodney Williams Memorial Foundation Research Training Grant (to PJA); the Science and Technology Research Program of the Liaoning Education Department (LK201647, to YD); the National Institute on Deafness and other Communication Disorders (NIDCD), NIH (F32DC014623, to EHN, and K08DC011043 and RO1DC01910); the Department of Defense (MR130316); the Akiko Yamazaki and Jerry Yang Faculty Scholar Fund; the California Initiative in Regenerative Medicine (RN3-06529, to AGC); and the Yu and Oberndorf families.

Address correspondence to: Alan G. Cheng, 801 Welch Road, Department of Otolaryngology-HNS, Stanford, California 94305, USA. Phone: 650.725.6500; Email: aglcheng@stanford.edu.
1. Avilion AA, Nicolis SK, Pevny LH, Perez L, Vivian $\mathrm{N}$, Lovell-Badge R. Multipotent cell lineages in early mouse development depend on SOX2 function. Genes Dev. 2003;17(1):126-140.

2. Masui S, et al. Pluripotency governed by Sox 2 via regulation of Oct $3 / 4$ expression in mouse embryonic stem cells. Nat Cell Biol. 2007;9(6):625-635.

3. Takahashi K, Yamanaka S. Induction of pluripotent stem cells from mouse embryonic and adult fibroblast cultures by defined factors. Cell. 2006;126(4):663-676.

4. $\mathrm{Yu} \mathrm{J}$, et al. Induced pluripotent stem cell lines derived from human somatic cells. Science. 2007;318(5858):1917-1920.

5. Bylund M, Andersson E, Novitch BG, Muhr J. Vertebrate neurogenesis is counteracted by Sox1-3 activity. Nat Neurosci. 2003;6(11):1162-1168.

6. Ellis P, et al. SOX2, a persistent marker for multipotential neural stem cells derived from embryonic stem cells, the embryo or the adult. Dev Neurosci. 2004;26(2-4):148-165.

7. Favaro R, et al. Hippocampal development and neural stem cell maintenance require Sox2-dependent regulation of Shh. Nat Neurosci. 2009;12(10):1248-1256.

8. Graham V, Khudyakov J, Ellis P, Pevny L. SOX2 functions to maintain neural progenitor identity. Neuron. 2003;39(5):749-765.

9. Hutton SR, Pevny LH. SOX2 expression levels distinguish between neural progenitor populations of the developing dorsal telencephalon. Dev Biol. 2011;352(1):40-47.

10. Taranova OV, et al. SOX2 is a dose-dependent regulator of retinal neural progenitor competence. Genes Dev. 2006;20(9):1187-1202.

11. Kiernan AE, et al. Sox 2 is required for sensory organ development in the mammalian inner ear. Nature. 2005;434(7036):1031-1035.

12. Driskell RR, Giangreco A, Jensen KB, Mulder KW, Watt FM. Sox2-positive dermal papilla cells specify hair follicle type in mammalian epidermis. Development. 2009;136(16):2815-2823.

13. Kamachi Y, Uchikawa M, Collignon J, Lovell-Badge $\mathrm{R}$, Kondoh H. Involvement of Sox1, 2 and 3 in the early and subsequent molecular events of lens induction. Development. 1998;125(13):2521-2532.

14. Okubo T, Clark C, Hogan BL. Cell lineage mapping of taste bud cells and keratinocytes in the mouse tongue and soft palate. Stem Cells.
2009;27(2):442-450.

15. Okubo T, Pevny LH, Hogan BL. Sox 2 is required for development of taste bud sensory cells. Genes Dev. 2006;20(19):2654-2659.

16. Que J, Luo X, Schwartz RJ, Hogan BL. Multiple roles for Sox 2 in the developing and adult mouse trachea. Development. 2009;136(11):1899-1907.

17. Que J, et al. Multiple dose-dependent roles for Sox 2 in the patterning and differentiation of anterior foregut endoderm. Development. 2007;134(13):2521-2531.

18. Fantes J, et al. Mutations in SOX2 cause anophthalmia. Nat Genet. 2003;33(4):461-463.

19. Kelberman D, et al. Mutations within Sox2/ SOX 2 are associated with abnormalities in the hypothalamo-pituitary-gonadal axis in mice and humans. J Clin Invest. 2006;116(9):2442-2455.

20. Sisodiya SM, et al. Role of SOX2 mutations in human hippocampal malformations and epilepsy. Epilepsia. 2006;47(3):534-542.

21. Arnold K, et al. Sox2(+) adult stem and progenitor cells are important for tissue regeneration and survival of mice. Cell Stem Cell. 2011;9(4):317-329.

22. Brazel CY, et al. Sox 2 expression defines a heterogeneous population of neurosphere-forming cells in the adult murine brain. Aging Cell. 2005;4(4):197-207.

23. Suh H, Consiglio A, Ray J, Sawai T, D’Amour $\mathrm{KA}$, Gage FH. In vivo fate analysis reveals the multipotent and self-renewal capacities of Sox $2+$ neural stem cells in the adult hippocampus. Cell Stem Cell. 2007;1(5):515-528.

24. Amador-Arjona A, et al. SOX 2 primes the epigenetic landscape in neural precursors enabling proper gene activation during hippocampal neurogenesis. Proc Natl Acad Sci US A. 2015;112(15):E1936-E1945.

25. Ferri AL, et al. Sox2 deficiency causes neurodegeneration and impaired neurogenesis in the adult mouse brain. Development. 2004;131(15):3805-3819.

26. Julian LM, et al. Opposing regulation of Sox 2 by cell-cycle effectors E2f3a and E2f3b in neural stem cells. Cell Stem Cell. 2013;12(4):440-452.

27. Gaete M, et al. Spinal cord regeneration in Xenopus tadpoles proceeds through activation of Sox2-positive cells. Neural Dev. 2012;7:13.

28. Kempfle JS, Turban JL, Edge AS. Sox2 in the differentiation of cochlear progenitor cells. Sci Rep.
2016;6:23293.

29. Woods C, Montcouquiol M, Kelley MW. Math1 regulates development of the sensory epithelium in the mammalian cochlea. Nat Neurosci. 2004;7(12):1310-1318.

30. Hertzano R, et al. Transcription profiling of inner ears from Pou4f3(ddl/ddl) identifies Gfi1 as a target of the Pou $4 \mathrm{f} 3$ deafness gene. Hum Mol Genet. 2004;13(18):2143-2153.

31. Vahava $\mathrm{O}$, et al. Mutation in transcription factor POU4F3 associated with inherited progressive hearing loss in humans. Science. 1998;279(5358):1950-1954.

32. Varfolomeev EE, et al. Targeted disruption of the mouse Caspase 8 gene ablates cell death induction by the TNF receptors, Fas/Apo1, and DR3 and is lethal prenatally. Immunity. 1998;9(2):267-276.

33. Hasson $\mathrm{T}$, et al. Unconventional myosins in inner-ear sensory epithelia. JCell Biol. 1997;137(6):1287-1307.

34. Oesterle EC, Campbell S, Taylor RR, Forge A, Hume CR. Sox 2 and JAGGED1 expression in normal and drug-damaged adult mouse inner ear. JAssoc Res Otolaryngol. 2008;9(1):65-89.

35. Dabdoub A, et al. Sox 2 signaling in prosensory domain specification and subsequent hair cell differentiation in the developing cochlea. Proc Natl Acad Sci U S A. 2008;105(47):18396-18401.

36. Ahmed M, Wong EY, Sun J, Xu J, Wang F, Xu PX. Eya1-Six1 interaction is sufficient to induce hair cell fate in the cochlea by activating Atoh1 expression in cooperation with Sox2. Dev Cell. 2012;22(2):377-390.

37. Ahmed M, Xu J, Xu PX. EYA1 and SIX1 drive the neuronal developmental program in cooperation with the SWI/SNF chromatin-remodeling complex and SOX2 in the mammalian inner ear. Development. 2012;139(11):1965-1977.

38. Puligilla C, Kelley MW. Dual role for Sox 2 in specification of sensory competence and regulation of Atoh1 function. Dev Neurobiol. 2017;77(1):3-13.

39. Pan W, Jin Y, Chen J, Rottier RJ, Steel KP, Kiernan AE. Ectopic expression of activated notch or SOX2 reveals similar and unique roles in the development of the sensory cell progenitors in the mammalian inner ear. J Neurosci. 2013;33(41):16146-16157.

40. Neves J, Parada C, Chamizo M, Giráldez F. Jagged 1 regulates the restriction of Sox 2 expression in 
the developing chicken inner ear: a mechanism for sensory organ specification. Development. 2011;138(4):735-744.

41. Neves J, Vachkov I, Giraldez F. Sox2 regulation of hair cell development: incoherence makes sense. Hear Res. 2013;297:20-29.

42. Ruben RJ, Van de Water T, Polesky A. Cell kinetics of the 11 and 12-day mouse otocysts. Laryngoscope. 1971;81(10):1708-1718.

43. Cox BC, et al. Spontaneous hair cell regeneration in the neonatal mouse cochlea in vivo. Development. 2014;141(4):816-829.

44. Tong $\mathrm{L}$, et al. Selective deletion of cochlear hair cells causes rapid age-dependent changes in spiral ganglion and cochlear nucleus neurons. JNeurosci. 2015;35(20):7878-7891.

45. Bramhall NF, Shi F, Arnold K, Hochedlinger K, Edge AS. Lgr5-positive supporting cells generate new hair cells in the postnatal cochlea. Stem Cell Reports. 2014;2(3):311-322.

46. Lin V, Golub JS, Nguyen TB, Hume CR, Oesterle EC, Stone JS. Inhibition of Notch activity promotes nonmitotic regeneration of hair cells in the adult mouse utricles. JNeurosci. 2011;31(43):15329-15339.

47. Wang GP, et al. Notch signaling and Atoh1 expression during hair cell regeneration in the mouse utricle. Hear Res. 2010;267(1-2):61-70.

48. Chen P, Johnson JE, Zoghbi HY, Segil N. The role of Math1 in inner ear development: Uncoupling the establishment of the sensory primordium from hair cell fate determination. Development. 2002;129(10):2495-2505.

49. Hume CR, Bratt DL, Oesterle EC. Expression of LHX3 and SOX2 during mouse inner ear development. Gene Expr Patterns. 2007;7(7):798-807.

50. Rose MF, et al. Math1 is essential for the development of hindbrain neurons critical for perinatal breathing. Neuron. 2009;64(3):341-354.

51. Cox BC, et al. Spontaneous hair cell regeneration in the neonatal mouse cochlea in vivo. Development. 2014;141(4):816-829.

52. Stamos JL, Weis WI. The $\beta$-catenin destruction complex. Cold Spring Harb Perspect Biol. 2013;5(1):a007898.

53. Harada N, et al. Intestinal polyposis in mice with a dominant stable mutation of the beta-catenin gene. EMBO J. 1999;18(21):5931-5942.

54. Chai $\mathrm{R}$, et al. Wnt signaling induces proliferation of sensory precursors in the postnatal mouse cochlea. Proc Natl Acad Sci U S A. 2012;109(21):8167-8172.

55. Shi F, Hu L, Edge AS. Generation of hair cells in neonatal mice by $\beta$-catenin overexpression in Lgr5-positive cochlear progenitors. Proc Natl Acad Sci U S A. 2013;110(34):13851-13856.

56. Behrens J, et al. Functional interaction of beta-catenin with the transcription factor LEF-1. Nature. 1996;382(6592):638-642.

57. Jan TA, et al. Tympanic border cells are Wnt-responsive and can act as progenitors for postnatal mouse cochlear cells. Development.
2013;140(6):1196-1206.

58 . Huth ME, et al. Designer aminoglycosides prevent cochlear hair cell loss and hearing loss. JClin Invest. 2015;125(2):583-592.

59. Cafaro J, Lee GS, Stone JS. Atoh1 expression defines activated progenitors and differentiating hair cells during avian hair cell regeneration. Dev Dyn. 2007;236(1):156-170.

60. Liu Z, et al. Regulation of p27Kip1 by Sox2 maintains quiescence of inner pillar cells in the murine auditory sensory epithelium. J Neurosci. 2012;32(31):10530-10540.

61. Millimaki BB, Sweet EM, Riley BB. Sox2 is required for maintenance and regeneration, but not initial development, of hair cells in the zebrafish inner ear. Dev Biol. 2010;338(2):262-269.

62. Nakamura Y, de Paiva Alves E, Veenstra GJ, Hoppler S. Tissue- and stage-specific Wnt target gene expression is controlled subsequent to $\beta$-catenin recruitment to cis-regulatory modules. Development. 2016;143(11):1914-1925.

63. Buchert M, et al. Genetic dissection of differential signaling threshold requirements for the Wnt/beta-catenin pathway in vivo. PLoS Genet. 2010;6(1):e1000816.

64. Kormish JD, Sinner D, Zorn AM. Interactions between SOX factors and Wnt/beta-catenin signaling in development and disease. Dev Dyn. 2010;239(1):56-68.

65. Sarkar A, et al. Sox2 Suppresses Gastric Tumorigenesis in Mice. Cell Rep. 2016;16(7):1929-1941.

66. Otsubo T, Akiyama Y, Yanagihara K, Yuasa Y. SOX2 is frequently downregulated in gastric cancers and inhibits cell growth through cell-cycle arrest and apoptosis. Br J Cancer. 2008;98(4):824-831.

67. Chen Y, et al. The molecular mechanism governing the oncogenic potential of SOX 2 in breast cancer. J Biol Chem. 2008;283(26):17969-17978.

68. Ni W, et al. Extensive supporting cell proliferation and mitotic hair cell generation by in vivo genetic reprogramming in the neonatal mouse cochlea.J Neurosci. 2016;36(33):8734-8745.

69. Abdolazimi Y, Stojanova Z, Segil N. Selection of cell fate in the organ of Corti involves the integration of Hes/Hey signaling at the Atoh1 promoter. Development. 2016;143(5):841-850.

70. Zine A, et al. Hes1 and Hes5 activities are required for the normal development of the hair cells in the mammalian inner ear. J Neurosci. 2001;21(13):4712-4720.

71. Doetzlhofer A, Basch ML, Ohyama T, Gessler M, Groves AK, Segil N. Hey2 regulation by FGF provides a Notch-independent mechanism for maintaining pillar cell fate in the organ of Corti. Dev Cell. 2009;16(1):58-69.

72. Maass JC, et al. Transcriptomic Analysis of Mouse Cochlear Supporting Cell Maturation Reveals Large-Scale Changes in Notch Responsiveness Prior to the Onset of Hearing. PLoS ONE. 2016;11(12):e0167286.

73. Yamamoto N, Tanigaki K, Tsuji M, Yabe D, Ito
J, Honjo T. Inhibition of Notch/RBP-J signaling induces hair cell formation in neonate mouse cochleas. J Mol Med. 2006;84(1):37-45.

74. Korrapati S, Roux I, Glowatzki E, Doetzlhofer A. Notch signaling limits supporting cell plasticity in the hair cell-damaged early postnatal murine cochlea. PLoS ONE. 2013;8(8):e73276.

75. Hartman BH, Basak O, Nelson BR, Taylor V, Bermingham-McDonogh O, Reh TA. Hes5 expression in the postnatal and adult mouse inner ear and the drug-damaged cochlea. JAssoc Res Otolaryngol. 2009;10(3):321-340.

76. Lanford PJ, Shailam R, Norton CR, Gridley T, Kelley MW. Expression of Math1 and HES5 in the cochleae of wildtype and Jag2 mutant mice. JAssoc Res Otolaryngol. 2000;1(2):161-171.

77. Maass JC, et al. Changes in the regulation of the Notch signaling pathway are temporally correlated with regenerative failure in the mouse cochlea. Front Cell Neurosci. 2015;9:110.

78. Doetzlhofer A, Avraham KB. Insights into inner ear-specific gene regulation: Epigenetics and non-coding RNAs in inner ear development and regeneration. Semin Cell Dev Biol. 2017;65:69-79.

79. Cox BC, Liu Z, Lagarde MM, Zuo J. Conditional gene expression in the mouse inner ear using CreloxP. J Assoc Res Otolaryngol. 2012;13(3):295-322.

80. Eagleson KL, et al. Disruption of Foxg1 expression by knock-in of cre recombinase: effects on the development of the mouse telencephalon. Neuroscience. 2007;148(2):385-399.

81. Shen L, Nam HS, Song P, Moore H, Anderson SA. FoxG1 haploinsufficiency results in impaired neurogenesis in the postnatal hippocampus and contextual memory deficits. Hippocampus. 2006;16(10):875-890.

82. Siegenthaler JA, Tremper-Wells BA, Miller MW. Foxg1 haploinsufficiency reduces the population of cortical intermediate progenitor cells: effect of increased p21 expression. Cereb Cortex. 2008;18(8):1865-1875.

83. Li W, et al. Notch inhibition induces mitotically generated hair cells in mammalian cochleae via activating the Wnt pathway. Proc Natl Acad Sci US A. 2015;112(1):166-171.

84. Avraham KB. Hear come more genes! Nat Med. 1998;4(11):1238-1239.

85. Young KM, Mitsumori T, Pringle N, Grist M, Kessaris N, Richardson WD. An Fgfr3-iCreER(T2) transgenic mouse line for studies of neural stem cells and astrocytes. Glia. 2010;58(8):943-953.

86. Madisen L, et al. A robust and high-throughput Cre reporting and characterization system for the whole mouse brain. Nat Neurosci. 2010;13(1):133-140.

87. Livak KJ, Schmittgen TD. Analysis of relative gene expression data using real-time quantitative PCR and the 2(-Delta Delta C(T)) Method. Methods. 2001;25(4):402-408.

88. Wang F, et al. RNAscope: a novel in situ RNA analysis platform for formalin-fixed, paraffin-embedded tissues. JMol Diagn. 2012;14(1):22-29. 